\title{
A new approach to the description of one-parameter groups of formal power series in one indeterminate
}

\author{
WoJCIECH JABŁOŃSKI AND LUDWIG REICH
}

\begin{abstract}
The aim of the paper is to describe one-parameter groups of formal power series, that is to find a general form of all homomorphisms $\Theta_{G}: G \rightarrow \Gamma, \Theta_{G}(t)=\sum_{k=1}^{\infty} c_{k}(t) X^{k}$, $c_{1}: G \rightarrow \mathbb{K} \backslash\{0\}, c_{k}: G \rightarrow \mathbb{K}$ for $k \geq 2$, from a commutative group $(G,+)$ into the group $(\Gamma, \circ)$ of invertible formal power series with coefficients in $\mathbb{K} \in\{\mathbb{R}, \mathbb{C}\}$. Considering one-parameter groups of formal power series and one-parameter groups of truncated formal power series, we give explicit formulas for the coefficient functions $c_{k}$ with more details in the case where either $c_{1}=1$ or $c_{1}$ takes infinitely many values. Here we give the results much more simply than they were presented in Jabłoński and Reich (Abh. Math. Sem. Univ. Hamburg 75:179-201, 2005; Result Math 47:61-68, 2005; Publ Math Debrecen 73(1-2):2547,2008 ). Also the case im $c_{1}=E_{m}$ (here $E_{m}$ stands for the group of all complex roots of order $m$ of 1), not considered in Jabłoński and Reich (Abh. Math. Sem. Univ. Hamburg 75:179-201, 2005; Result Math 47:61-68, 2005; Publ Math Debrecen 73(1-2):25-47, 2008), will be discussed.
\end{abstract}

Mathematics Subject Classification (1991). Primary 39B72, 13F25; Secondary 13J05, 13H05.

Keywords. Formal power series, Translation equation.

\section{Contents}

1. Introduction 248

2. The ring of formal power series 250

3. Properties of the group operations in $\Gamma^{s} \quad 251$

4. One-parameter groups of formal power series 255

5. The regular one-parameter groups of formal power series 257

6. Properties of the sequences $\left(L_{n}\right)$ and $\left(P_{n}\right) \quad 262$

7. One-parameter groups in cases 1. and 3. 265

8. One-parameter groups in case $2 . \quad 271$

9. Iterative roots of unit in $\Gamma^{s} \quad 276$

10. One-parameter groups with infinite image 276

11. Embedding a power series into a one-parameter group 282

Open Access 283

$\begin{array}{ll}\text { References } & 284\end{array}$ 


\section{Introduction}

Let $\Gamma$ denote the group of all invertible formal power series in one indeterminate with substitution $\circ$ as a binary operation. By a one-parameter group of formal power series (FPS) we mean any homomorphism $\Theta_{G}$ from a group $(G,+)$ into $(\Gamma, \circ)$, i.e. any function $\Theta_{G}: G \rightarrow \Gamma$ satisfying

$$
\Theta_{G}\left(t_{1}+t_{2}\right)(X)=\left(\Theta_{G}\left(t_{1}\right) \circ \Theta_{G}\left(t_{2}\right)\right)(X) \text { for } t_{1}, t_{2} \in G .
$$

Analogously, we may define a one-parameter group of $s$-truncated FPS as a homomorphism $\Theta_{G}^{s}: G \rightarrow \Gamma^{s}$ into the group $\Gamma^{s}$ of all invertible $s$-truncated FPS in one indeterminate.

Let $F_{t}(X)=F(t, X):=\Theta_{G}(t)(X)$ and $F_{t}^{[s]}(X)=F^{[s]}(t, X):=\Theta_{G}^{s}(t)(X)$. If $\Theta_{G}: G \rightarrow \Gamma$ and $\Theta_{G}^{s}: G \rightarrow \Gamma^{s}$ are, a one-parameter group of FPS and a one-parameter group of $s$-truncated FPS, respectively, we will also say that the families $\left(F_{t}(X)\right)_{t \in G}$ and $\left(F_{t}^{[s]}(X)\right)_{t \in G}$ are a one-parameter group of FPS and a one-parameter group of $s$-truncated FPS. In this case the families $\left(F_{t}(X)\right)_{t \in G}$ and $\left(F_{t}^{[s]}(X)\right)_{t \in G}$ satisfy the well known translation equation

$$
\left\{\begin{array}{l}
F\left(t_{1}+t_{2}, X\right)=F\left(t_{1}, F\left(t_{2}, X\right)\right) \quad \text { for } t_{1}, t_{2} \in G, \\
F(0, X)=X
\end{array}\right.
$$

in the ring of FPS, and

$$
\left\{\begin{array}{l}
F^{[s]}\left(t_{1}+t_{2}, X\right)=F^{[s]}\left(t_{1}, F^{[s]}\left(t_{2}, X\right)\right) \bmod X^{s+1} \quad \text { for } t_{1}, t_{2} \in G, \\
F^{[s]}(0, X)=X,
\end{array}\right.
$$

in the ring of $s$-truncated FPS. Both of them may be written (with $F^{[\infty]}:=F$ ) in a unified way as

$$
\left\{\begin{array}{l}
F_{t_{1}+t_{2}}^{[s]}=F_{t_{1}}^{[s]} \circ F_{t_{2}}^{[s]} \quad \text { for } t_{1}, t_{2} \in G, \\
F_{0}^{[s]}=\mathrm{id} .
\end{array}\right.
$$

It appears that the form of both one-parameter groups $\Theta_{G}$ and $\Theta_{G}^{s}$ strongly depend on the function $c_{1}$. We will see that $c_{1}$ must be an exponential function, i.e. a homomorphism of $(G,+)$ into $(\mathbb{K} \backslash\{0\}, \cdot)$. By the first isomorphism theorem we know that $G / \operatorname{ker} c_{1} \cong \operatorname{im} c_{1}$. There appear the following cases:

case 1. $c_{1}=1$, which means that $\operatorname{ker} c_{1}=G$; then $\Theta_{G}$ and $\Theta_{G}^{s}$ have a rather simple structure;

case 2. $c_{1} \neq 1$, but $G / \operatorname{ker} c_{1} \cong \operatorname{im} c_{1}$ is a finite subgroup of $(\mathbb{K} \backslash\{0\}, \cdot)$; then im $c_{1}=E_{m}$ with some integer $m \geq 2$, where $E_{m}$ denotes the set of all roots of 1 of order $m$, and the general structure of such one-parameter groups is much more complicated;

case 3. $G / \operatorname{ker} c_{1} \cong \operatorname{im} c_{1}$ is an infinite subgroup of $(\mathbb{K} \backslash\{0\}, \cdot)$, then the general form of $\Theta_{G}$ and $\Theta_{G}^{s}$ is also simple. 
In order to solve our problem we use algebraic methods jointly with some tools from differential equations and functional equations. We begin with properties of the operation of substitution in the groups $\Gamma$ and $\Gamma^{s}$, which are crucial for our method. Then we find (Theorem 1) regular one-parameter groups of formal power series, i.e. groups with coefficients being $C^{\infty}$-functions in the real case and entire when $\mathbb{K}=\mathbb{C}$.

Case 1 and case 3 were already investigated in $[6,7,9]$ using connections with the differential groups $L_{s}^{1}(s \in \mathbb{N})$ and $L_{\infty}^{1}$. These differential groups will not appear in the present paper. However we will come back to our previous results in cases 1 and 3 since we are now able to give new and simple proofs (see e.g. Theorems 4 and 5). The new idea consists in constructing a particular solution of inhomogeneous functional equations like (33) and (38) using the polynomials obtained in the representations of regular solutions of the translation equation and then adding the simple general solution of the corresponding homogeneous equation, like the Cauchy equation or (35). Our proofs for results such as Theorems 4 and 5 in our previous publications were considerably longer since we had to transfer certain results on differential groups to the groups of invertible formal power series and back.

Case 3 and a subcase of case 2 were already treated in [8] where the standard form of the solution was given whenever it can be applied. Here we give a description of the solutions in this subcase of case 2 using a sequence of polynomials for the representation of the solution (see Corollary 6), but the proof differs in some details (see the functional equation (48)).

Completely new in the present paper is Sect. 10. We give a solution in the following subcase of case 2, namely where we assume that $\Theta(G)$ is infinite and the set $\left\{l t_{0} \in G: 0 \leq l \leq m-1\right\}$ is a subgroup of $G$ and $t_{0} \in G$ is such that $c_{1}\left(t_{0}\right)$ is a primitive $m$ th root of 1 (Theorem 6 ).

As an application of our results we deal with the embedding problems (Sect. 11), which have so far been only studied related to homomorphisms from the group $(\mathbb{C},+)$ into $\Gamma$.

At the end of the introduction we would like to mention that our results contain the description of all one-parameter groups $\Theta: G \rightarrow \Gamma^{s}$ for such groups $G$ as $(\mathbb{R},+),(\mathbb{C},+),\left(\mathbb{R}^{*}, \cdot\right)$ and $\left(\mathbb{C}^{*}, \cdot\right)$.

It seems that our methods of combining functional equations, differential equations and algebraic methods will not give a satisfactory solution of our problem (i.e. construction of one-parameter groups of FPS) in the remaining subcase. A possible approach to cover also this last open case could come from a theory of families of commuting invertible FPS.

The description of such one-parameter groups uses some sequences of polynomials. Using properties of these sequences (Lemmas 4 and 5), we describe the general form of one-parameter groups in cases 1 . and 3 for both FPS and $s$-truncated FPS. Finally, we consider the second case. In the subcase when one-parameter groups are finite, we give the explicit form of the coefficient 
functions of these groups. Also a subcase where $c_{1}(G)$ is finite and $\Theta(G)$ is infinite is considered.

\section{The ring of formal power series}

$\mathbb{K} \llbracket X \rrbracket$ will denote the ring of all formal power series (FPS) $\sum_{k=0}^{\infty} c_{k} X^{k}$ with coefficients $c_{k} \in \mathbb{K}$, where $\mathbb{K} \in\{\mathbb{R}, \mathbb{C}\}$ is the field of real or complex numbers. For a formal power series $f(X)=\sum_{k=0}^{\infty} c_{k} X^{k}$ with $c_{i} \neq 0$ for some $i \in \mathbb{N} \cup\{0\}$ $(\mathbb{N}$ stands here for the set of all positive integers) we define

$$
\text { ord } f(X)=\min \left\{i \in \mathbb{N} \cup\{0\}: c_{i} \neq 0\right\},
$$

assuming additionally ord $\left(\sum_{k=0}^{\infty} 0 X_{k}\right)=\infty$.

The set $\Gamma=\{f(X) \in \mathbb{K} \llbracket X \rrbracket:$ ord $f(X)=1\}$ with the substitution $\circ$ as a binary operation is a group. Moreover, the set $\Gamma_{1}=$ $\left\{\sum_{k=1}^{\infty} c_{k} X^{k} \in \Gamma: c_{1}=1\right\}$ is a subgroup of $\Gamma$. A very good reference for this topic is [2].

In the sequel we will need the notion of the ring of truncated formal power series. It is known that for a fixed $s \in \mathbb{N}$ the set

$$
I_{s}=X^{s+1} \mathbb{K} \llbracket X \rrbracket=\{f(X) \in \mathbb{K} \llbracket X \rrbracket: \text { ord } f(X) \geq s+1\}
$$

is an ideal in the ring $\mathbb{K} \llbracket X \rrbracket$. We may then define a congruence modulo $X^{s+1}$ as follows: we say that $f_{1}(X), f_{2}(X) \in \mathbb{K} \llbracket X \rrbracket$ are congruent modulo $X^{s+1}$ (which will be written as $\left(f_{1}(X) \equiv f_{2}(X) \bmod X^{s+1}\right)$ provided $\left(f_{1}-f_{2}\right)(X)=$ $f_{1}(X)-f_{2}(X) \in I_{s}$. This means that $X^{s+1}$ is a divisor of the difference $\left(f_{1}-f_{2}\right)(X)$.

We consider the quotient ring $\mathbb{K} \llbracket X \rrbracket / I_{s}$ of all cosets $[f(X)]_{s}=f(X)+I_{s}$. With every coset $f(X)+I_{s}$, where $f(X)=\sum_{k=0}^{\infty} c_{k} X^{k} \in \mathbb{K} \llbracket X \rrbracket$, we may associate an $s$-truncation of a formal power series $f(X)$ defined by

$$
f^{[s]}(X):=\sum_{k=0}^{s} c_{k} X^{k} \in \mathbb{K} \llbracket X \rrbracket_{s} \subset \mathbb{K}[X] \subset \mathbb{K} \llbracket X \rrbracket .
$$

In the set $\mathbb{K} \llbracket X \rrbracket_{s}$ (which may be treated as a set of all polynomials of degree at most $s$ ) we introduce, in a natural way, an addition of truncated formal power series. It appears that a multiplication and a substitution must be defined in a specific way so that $\mathbb{K} \llbracket X \rrbracket_{s}$ should be closed under them. Let for $f(X), g(X) \in \mathbb{K} \llbracket X \rrbracket_{s}$,

$$
(f g)(X):=(f g)^{[s]}(X),
$$

and, in the case when ord $g(X) \geq 1$,

$$
(f \circ g)(X):=(f \circ g)^{[s]}(X) .
$$

Then $\left(\mathbb{K} \llbracket X \rrbracket_{s},+, \cdot\right)$ is a ring which is isomorphic to $\mathbb{K} \llbracket X \rrbracket / I_{s}$. Moreover, the set $\Gamma^{s}:=\left\{f(X) \in \mathbb{K} \llbracket X \rrbracket_{s}\right.$ : ord $\left.f(X)=1\right\}$ is a group under substitution. 
We will also need the notion of a semicanonical form of a formal power series in $\Gamma$ and $\Gamma^{s}$. Namely, for a fixed integer $m \geq 1$, let $\mathcal{N}_{m}$ be the set of all $f(X) \in \Gamma$ such that $f(X)=\sum_{k=0}^{\infty} c_{k m+1} X^{k m+1}$, whereas $\mathcal{N}_{m}^{s}$ stands for the set of all $f(X) \in \Gamma^{s}$ such that $f(X)=\sum_{k=0}^{r} c_{k m+1} X^{k m+1}$, where $r m+1 \leq s<(r+1) m+1$.

To unify our considerations for both FPS and $s$-truncated FPS we put $\Gamma^{\infty}:=\Gamma$ and $\mathcal{N}_{m}^{\infty}:=\mathcal{N}_{m}$. It is known that if $\rho \in E_{m}$ is a primitive root of 1 of order $m$ and $L_{\rho}(X)=\rho X$ then for both $s$ being a positive integer and $s=\infty$ we have

$$
\mathcal{N}_{m}^{s}=\left\{f(X) \in \Gamma^{s}:\left(f \circ L_{\rho}\right)(X)=\left(L_{\rho} \circ f\right)(X)\right\} .
$$

Thus $\mathcal{N}_{m}^{s}$ is a subgroup of $\Gamma^{s}$.

\section{Properties of the group operations in $\Gamma^{s}$}

We give here a crucial formula describing the operation of substitution $\circ$ in the group $\Gamma^{\infty}$. Because of the construction of $\Gamma^{s}$ the same formula is also valid in $\Gamma^{s}$. Let $|k, l|$ denote the set of all integers $n$ such that $k \leq n \leq l$, and let $|k, \infty|$ be the set of all integers $n \geq k$. We assume that $\sum_{t \in \emptyset} a_{t}=0$ and $\prod_{t \in \emptyset} a_{t}=1$. For a fixed integer $r \geq 1$, let $\mathbb{N}_{r}=\{l r+1: l \in \mathbb{N} \cup\{0\}\}$. In particular $\mathbb{N}_{1}=\mathbb{N}$.

Let $\sum_{k=1}^{\infty} a_{k} X^{k}, \sum_{k=1}^{\infty} b_{k} X^{k} \in \Gamma^{\infty}$. It is known (cf. [6]) that if

$$
\sum_{k=1}^{\infty} a_{k}\left(\sum_{l=1}^{\infty} b_{l} X^{l}\right)^{k}=\sum_{n=1}^{\infty} d_{n} X^{n}
$$

then

$$
d_{n}=\sum_{k=1}^{n} a_{k} \sum_{\bar{u}_{n} \in U_{n, k}} B_{\bar{u}_{n}} \prod_{j=1}^{n} b_{j}^{u_{j}} \quad \text { for } n \in \mathbb{N},
$$

where

$$
\begin{aligned}
& U_{n, k}:=\left\{\bar{u}_{n}:=\left(u_{1}, \ldots, u_{n}\right) \in|0, k|^{n}: \sum_{j=1}^{n} u_{j}=k, \sum_{j=1}^{n} j u_{j}=n\right\}, \\
& B_{\bar{u}_{n}}:=\frac{k !}{\prod_{j=1}^{n} u_{j} !} .
\end{aligned}
$$

Note that $B_{\bar{u}_{n}}$ is a multinomial coefficient. As examples of (4) we quote

$$
d_{1}=a_{1} b_{1}, \quad d_{2}=a_{1} b_{2}+a_{2} b_{1}^{2}, \quad d_{3}=a_{1} b_{3}+2 a_{2} b_{1} b_{2}+a_{3} b_{1}^{3} .
$$

Now, for the convenience of the reader we collect several properties of formulas (4). Although these properties are similar to those of the binary operation in the Lie group $L_{s}^{1}$ (cf. [6, Theorem 2]), for the completeness of the paper, 
we give their proofs here. We mention in the paper, when such a detailed property is needed in a proof, and the reader may postpone the study of these properties until they really appear. Let for an integer $p \geq 0$

$$
U_{n, k}^{p}:=\left\{\bar{u}_{n} \in U_{n, k}: \forall_{j \in|2, p+1|, j \leq n-1} u_{j}=0\right\} .
$$

Clearly $U_{n, k}^{p_{2}} \subset U_{n, k}^{p_{1}} \subset U_{n, k}^{0}=U_{n, k}$ for $0 \leq p_{1} \leq p_{2}$. Moreover, we put $U_{n, k}^{1,1}=U_{n, k}$, and, for $p \geq 1, p+1=q m$ with some integers $m \geq 2, q \geq 1$, we define

$$
U_{n, k}^{m, q}:=\left\{\bar{u}_{n} \in U_{n, k}: \forall_{j \in|2, q m| \cup\left(|q m+2, n| \backslash \mathbb{N}_{m}\right)} u_{j}=0\right\} .
$$

Then $U_{n, k}^{m, q} \subset U_{n, k}^{p}$. We begin with properties of the sets $U_{n, k}, U_{n, k}^{p}$ and $U_{n, k}^{m, q}$.

Lemma 1. (cf. $[1,4,5]$ ) Let $n \in|2, \infty|$ be arbitrarily fixed. Then

(ii) $B_{\bar{u}_{n}}=1$ for $\bar{u}_{n} \in U_{n, 1} \cup U_{n, n}$;

(iii) if $n \geq 3, k \in|2, n-1|$ and $\bar{u}_{n} \in U_{n, k}$, then $u_{j}=0$ for every $j \in$ $|n-k+2, n|$ and there exists $j \in|2, n-k+1|$ with $u_{j} \geq 1$;

(iv) if $p \geq 1$ is an integer, $n \geq p+3$ and $k \in|n-p, n-1|$, then $U_{n, k}^{p}=\emptyset$;

(v) if $m \geq 2, q \geq 1$ and $n \in \mathbb{N} \backslash \mathbb{N}_{m}$, then for each $k \in|q m+1, n-q m| \cap \mathbb{N}_{m}$ we have $U_{n, k}^{m, q}=\emptyset$.

Proof. The properties (i)-(ii) are simple consequences of the conditions defining the set $U_{n, k}$.

(iii) If for $k \in|2, n-1|$ and $\bar{u}_{n} \in U_{n, k}$ we had $u_{l} \neq 0$ for some $l \in|n-k+2, n|$, then, by the conditions defining $U_{n, k}$ we would obtain

$$
n-k=\sum_{j=1}^{n} j u_{j}-\sum_{j=1}^{n} u_{j}=\sum_{j=2}^{n}(j-1) u_{j} \geq(l-1) u_{l} \geq n-k+1,
$$

which leads to a contradiction. Further, suppose that $u_{j}=0$ for all $j \in \mid 2, n-$ $k+1 \mid$. Since also $u_{j}=0$ for $j \in|n-k+2, n|$, then $k=\sum_{i=1}^{n} u_{i}=u_{1}=$ $\sum_{i=1}^{n} i u_{i}=n$, which is impossible, so there exists $j \in|2, n-k+1|$ with $u_{j} \geq 1$.

(iv) Let $p \geq 1$ be an integer, $n \geq p+3, k \in|n-p, n-1|$. Suppose that $U_{n, k}^{p} \neq \emptyset$. By (iii), for $\bar{u}_{n} \in U_{n, k}^{p}$ there exists then $j \in|2, n-k+1| \subset|2, p+1|$ such that $u_{j} \geq 1$, which leads to a contradiction with $\bar{u}_{n} \in U_{n, k}^{p}$. Hence $U_{n, k}^{p}=\emptyset$.

(v) Let $n \in \mathbb{N} \backslash \mathbb{N}_{m}, k \in|p+2, n-p-1| \cap \mathbb{N}_{m}$ and fix $\bar{u}_{n} \in U_{n, k}^{m, q}$. For every $j \in|2, n-k+1| \backslash \mathbb{N}_{m}$ we have $u_{j}=0$. Then $n-k=\sum_{j=2}^{n}(j-1) u_{j}=$ $\sum_{j \in|2, n-k+1| \cap \mathbb{N}_{m}}(j-1) u_{j}$, which gives

$$
n=k+\sum_{j \in|2, n-k+1| \cap \mathbb{N}_{m}}(j-1) u_{j} \in \mathbb{N}_{m} .
$$

This contradiction finishes the proof. 
When studying one-parameter groups of FPS we will frequently use the following special form of (4).

Corollary 1. (cf. [5, Lemma 2]) Let $p \in|0, \infty|$ and assume in (4) that $a_{j}=$ $b_{j}=0$ for $j \in|2, p+1|$. Then $d_{n}=0$ for $n \in|2, p+1|$ and

$$
d_{n}=a_{1} b_{n}+\sum_{k=p+2}^{n-p-1} a_{k} \sum_{\bar{u}_{n} \in U_{n, k}^{p}} B_{\bar{u}_{n}} b_{1}^{u_{1}} \prod_{j=p+2}^{n-k+1} b_{j}^{u_{j}}+a_{n} b_{1}^{n} \quad \text { for } n \in|p+2, \infty| .
$$

Proof. For every $n \geq 2$, by Lemma 1 (i)-(iii), we have

$$
d_{n}=a_{1} b_{n}+\sum_{k=2}^{n-1} a_{k} \sum_{\bar{u}_{n} \in U_{n, k}} B_{\bar{u}_{n}} b_{1}^{u_{1}} \prod_{j=2}^{n-k+1} b_{j}^{u_{j}}+a_{n} b_{1}^{n}
$$

Hence, for $n \in|2, p+1|$ we have $d_{n}=0$ and $d_{p+2}=a_{1} b_{p+2}+a_{p+2} b_{1}^{p+2}$, which gives (6) for $n=p+2$. Now, let $n \geq p+3$. We have

$$
d_{n}=a_{1} b_{n}+\sum_{k=p+2}^{n-1} a_{k} \sum_{\bar{u}_{n} \in U_{n, k}} B_{\bar{u}_{n}} b_{1}^{u_{1}} \prod_{j=2}^{n-k+1} b_{j}^{u_{j}}+a_{n} b_{1}^{n} .
$$

Note that for every $k \in|p+2, n-1|$ we have

$$
\sum_{\bar{u}_{n} \in U_{n, k}} B_{\bar{u}_{n}} b_{1}^{u_{1}} \prod_{j=2}^{n-k+1} b_{j}^{u_{j}}=\sum_{\bar{u}_{n} \in U_{n, k}^{p}} B_{\bar{u}_{n}} b_{1}^{u_{1}} \prod_{j=p+2}^{n-k+1} b_{j}^{u_{j}} .
$$

In the case when $p \geq 1$, for $k \in|n-p, n-1|$, because of Lemma 1 (iv) we get $U_{n, k}^{p}=\emptyset$. Then from (7) we obtain (6), which finishes the proof.

The following very detailed properties of (4) will be useful in the construction of a one-parameter group of FPS.

Corollary 2. Let $p \geq 1, p+1=q m$ with integers $m \geq 2$ and $q \geq 1$. If $a_{j}=b_{j}=0$ for $j \in|2, p+1|$ and $b_{j}=0$ for $j \in|p+3, \infty| \backslash \mathbb{N}_{m}$, then for $n \in|p+2, \infty|$

$$
d_{n}=a_{1} b_{n}+\sum_{k=p+2}^{n-p-1} a_{k} \sum_{\bar{u}_{n} \in U_{n, k}^{m, q}} B_{\bar{u}_{n}} b_{1}^{u_{1}} \prod_{j \in|p+2, n-k+1| \cap \mathbb{N}_{m}} b_{j}^{u_{j}}+a_{n} b_{1}^{n} .
$$

Proof. Since $b_{i}=0$ for $i \in|p+3, \infty| \backslash \mathbb{N}_{m}, \sum_{\bar{u}_{n} \in U_{n, k}^{p} \backslash U_{n, k}^{m, q}} B_{\bar{u}_{n}} b_{1}^{u_{1}} \prod_{j=p+2}^{n-k+1} b_{j}^{u_{j}}$ $=0$ for every $k \in|p+2, n-p-1|$. Hence (6) gives (8).

Corollary 3. Let $p \geq 1, p+1=q m$ with integers $m \geq 2, q \geq 1$, and let $a_{j}=b_{j}=0$ for $j \in|2, p+1| \cup\left(|p+3, \infty| \backslash \mathbb{N}_{m}\right)$. Then 
(i) $\quad$ for $n \in|p+3, \infty| \backslash \mathbb{N}_{m}$

$$
\sum_{k=p+2}^{n-p-1} a_{k} \sum_{\bar{u}_{n} \in U_{n, k}^{m, q}} B_{\bar{u}_{n}} b_{1}^{u_{1}} \prod_{j \in|p+2, n-k+1| \cap \mathbb{N}_{m}} b_{j}^{u_{j}}=0
$$

hence $d_{n}=0$,

(ii) for $n=r m+1 \in|p+2, \infty| \cap \mathbb{N}_{m}$

$$
\begin{aligned}
d_{r m+1}= & a_{1} b_{r m+1}+\sum_{k=q}^{r-q} a_{k m+1} \sum_{\bar{u}_{r m+1} \in U_{r m+1, k m+1}^{m, q}} B_{\bar{u}_{n}} b_{1}^{u_{1}} \prod_{j=q}^{r-k} b_{j m+1}^{u_{j m+1}} \\
& +a_{r m+1} b_{1}^{r m+1} .
\end{aligned}
$$

Proof. (i) If $n \in|p+3, \infty| \backslash \mathbb{N}_{m}$, then for $k \in|p+2, n-p-1| \backslash \mathbb{N}_{m}$ we have $a_{k}=0$, and for $k \in|p+2, n-p-1| \cap \mathbb{N}_{m}$, by Lemma $1(\mathrm{v}), U_{n, k}^{m, q}=\emptyset$.

This implies (9), and, in consequence, (8) gives $d_{n}=0$.

(ii) (10) is a simple consequence of the assumptions and (8).

Further properties of (4) will be useful in the proof of some properties of sequences of the polynomials $\left(L_{n}^{p+2}\right)$ and $\left(P_{n}\right)$ which appear in the description of one-parameters groups of FPS. To simplify the notation we put

$$
(\alpha, \ldots, \beta, \stackrel{k}{x}, \gamma, \ldots, \delta)
$$

in order to identify $x$ as the $k$ th element of the sequence $(\alpha, \ldots, \beta, x, \gamma, \ldots, \delta)$.

Lemma 2. Let $p \geq 0, n \in|p+3, \infty|$. Then

$$
\begin{aligned}
U_{n+p+1, n}^{p}= & \{(n-1,0, \ldots, 0, \stackrel{p+2}{1}, 0, \ldots, 0)\}, \\
U_{n+p+1, p+2}^{p}= & \{(p+1,0, \ldots, 0, \stackrel{n}{1}, 0, \ldots, 0)\} \\
& \cup\left\{\bar{u}_{n+p+1} \in U_{n+p+1, p+2}^{p}: \forall_{j \in|n, n+p+1|} u_{j}=0\right\} .
\end{aligned}
$$

For $\bar{u}_{n+p+1}=(p+1,0, \ldots, 0, \stackrel{n}{1}, 0, \ldots, 0) \in U_{n+p+1, p+2}^{p}$ we have $B_{\bar{u}_{n+p+1}}=$ $p+2$, and, for $\bar{u}_{n+p+1}=(n-1,0, \ldots, 0, \stackrel{p+2}{1}, 0, \ldots, 0) \in U_{n+p+1, n}^{p}$, $B_{\bar{u}_{n+p+1}}=n$.

Proof. Let $u_{n+p+1}=\left(u_{1}, \ldots, u_{n+p+1}\right) \in U_{n+p+1, n}^{p} \subset U_{n+p+1, n}$. Then, by the description of $U_{n, k}$ we get $p+1=n+p+1-n=\sum_{j=2}^{n+p+1}(j-1) u_{j}$, which, jointly with $u_{j}=0$ for $j \in|2, p+1|$, gives $u_{p+2}=1$ and $u_{j}=0$ for $j \in|p+3, n+p+1|$. Thus $u_{1}=n-1$. 
Next, let $u_{n+p+1}=\left(u_{1}, \ldots, u_{n+p+1}\right) \in U_{n+p+1, p+2}^{p}$. Then

$$
n-1=n+p+1-(p+2)=\sum_{j=2}^{n+p+1}(j-1) u_{j} .
$$

If $u_{n}=1$, then $u_{j}=0$ for every $j \in|2, n+p+1| \backslash\{n\}$, hence $u_{1}=p+1$. Otherwise, $u_{n}=0$ and, by Lemma 1 (iv), we also get $u_{j}=0$ for $j \in \mid n+p+$ $1-(p+2)+2, n+p+1|=| n+1, n+p+1 \mid$, which finishes the proof of the decomposition of the set $U_{n+p+1, p+2}^{p}$.

The last statement follows from the equality defining $B_{\bar{u}_{n+p+1}}$.

Now, let for $n \in|p+3, \infty|$,

$$
\begin{aligned}
\widehat{U}_{n+p+1, p+2}^{p} & =\left\{\bar{u}_{n+p+1} \in U_{n+p+1, p+2}^{p}: \forall_{j \in|n, n+p+1|} u_{j}=0\right\}, \\
\widehat{U}_{n+p+1, k}^{p} & =U_{n+p+1, k}^{p} \quad \text { for } k \in|p+3, n-p-1| .
\end{aligned}
$$

Then, for each $k \in|p+2, n-p-1|$ and every $u_{n+p+1} \in \widehat{U}_{n+p+1, k}^{p}$ we have $u_{j}=0$ for $j \in|n, n+p+1|$. From Corollary 1 and Lemma 2 we obtain

Corollary 4. Let $p \geq 0$. If $a_{j}=b_{j}=0$ for all $j \in|2, p+1|$, then for $n \in|p+2, \infty|$ with $r=n+p+1$ we have

$$
\begin{aligned}
d_{r}= & a_{1} b_{r}+(p+2) a_{p+2} b_{1}^{p+1} b_{n}+\sum_{k=p+2}^{n-1} a_{k} \sum_{\bar{u}_{r} \in \widehat{U}_{r, k}^{p}} B_{\bar{u}_{r}} b_{1}^{u_{1}} \prod_{j=p+2}^{r-k+1} b_{j}^{u_{j}} \\
& +n a_{n} b_{1}^{n-1} b_{p+2}+a_{r} b_{1}^{r}
\end{aligned}
$$

and $\sum_{k=p+2}^{n-1} a_{k} \sum_{\bar{u}_{r} \in \widehat{U}_{r, k}^{p}} B_{\bar{u}_{r}} b_{1}^{u_{1}} \prod_{j=p+2}^{r-k+1} b_{j}^{u_{j}}$ does not contain $a_{k}, b_{k}$ for $k \in$ $|n, r|$.

\section{One-parameter groups of formal power series}

Let $F(t, X)=\sum_{k=1}^{\infty} c_{k}(t) X^{k}$, where $c_{1}: G \rightarrow \mathbb{K} \backslash\{0\}, c_{k}: G \rightarrow \mathbb{K}$ for $k \in|2, \infty|$, be a one-parameter group of FPS. Then, from (1) we get

$$
\sum_{n=1}^{\infty} c_{n}\left(t_{1}+t_{2}\right) X^{n}=\sum_{l=1}^{\infty} c_{l}\left(t_{1}\right)\left(\sum_{j=1}^{\infty} c_{j}\left(t_{2}\right) X^{j}\right)^{l} \quad \text { for } t_{1}, t_{2} \in G
$$

Hence, on account of (5) and Corollary 1 with $p=0$, by comparing coefficients, we obtain the infinite system of functional equations 


$$
\left\{\begin{array}{l}
c_{1}\left(t_{1}+t_{2}\right)=c_{1}\left(t_{1}\right) c_{1}\left(t_{2}\right) \\
c_{2}\left(t_{1}+t_{2}\right)=c_{1}\left(t_{1}\right) c_{2}\left(t_{2}\right)+c_{2}\left(t_{1}\right) c_{1}\left(t_{2}\right)^{2} \\
\vdots \\
c_{n}\left(t_{1}+t_{2}\right)=c_{1}\left(t_{1}\right) c_{n}\left(t_{2}\right) \\
\quad+\sum_{k=2}^{n-1} c_{k}\left(t_{1}\right) \sum_{\bar{u}_{n} \in U_{n, k}} B_{\bar{u}_{n}} \prod_{j=1}^{n-k+1} c_{j}\left(t_{2}\right)^{u_{j}}+c_{n}\left(t_{1}\right) c_{1}\left(t_{2}\right)^{n} \\
\vdots
\end{array}\right.
$$

for $t_{1}, t_{2} \in G$.

In the same way, with $F^{[s]}(t, X)=\sum_{k=1}^{s} c_{k}(t) X^{k}$ being a one-parameter group of $s$-truncated FPS, where $c_{1}: G \rightarrow \mathbb{K} \backslash\{0\}, c_{k}: G \rightarrow \mathbb{K}$ for $k \in|2, s|$, we obtain from $(2)$

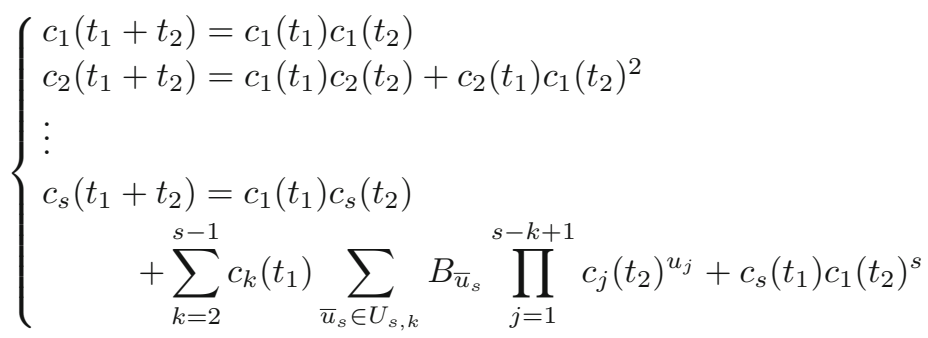

for $t_{1}, t_{2} \in G$. Note that in both cases $c_{1}$ must be a generalized exponential function.

Considering at the same time both $s \in \mathbb{N}$ and $s=\infty$, we will generally distinguish two cases, $c_{1}=1$ and $c_{1} \neq 1$ (in fact, the second one will be divided into subcases later on). In the first case we have clearly the trivial solution $c_{1}=1$ and $c_{n}=0$ for $n \in|2, s|$. So, in the case $c_{1}=1$, passing over the trivial case, without loss of generality we may assume that there is a nonnegative integer $p$ with $p+2 \leq s$ such that $c_{j}=0$ for $j \in|2, p+1|$ and $c_{p+2} \neq 0$. Then, by Corollary 1 , the systems (11) and (12) may be written as

$$
\left\{\begin{array}{l}
c_{p+2}\left(t_{1}+t_{2}\right)=c_{p+2}\left(t_{1}\right)+c_{p+2}\left(t_{2}\right) \\
c_{n}\left(t_{1}+t_{2}\right)=c_{n}\left(t_{1}\right) \\
\quad+\sum_{k=p+2}^{n-p-1} c_{k}\left(t_{1}\right) \sum_{\bar{u}_{n} \in U_{n, k}^{p}} B_{\bar{u}_{n}} \prod_{j=p+2}^{n-k+1} c_{j}\left(t_{2}\right)^{u_{j}}+c_{n}\left(t_{2}\right), \quad n \in|p+3, s|
\end{array}\right.
$$

for $t_{1}, t_{2} \in G$. In the second case we consider the system 


$$
\left\{\begin{array}{l}
c_{1}\left(t_{1}+t_{2}\right)=c_{1}\left(t_{1}\right) c_{1}\left(t_{2}\right) \\
c_{2}\left(t_{1}+t_{2}\right)=c_{1}\left(t_{1}\right) c_{2}\left(t_{2}\right)+c_{2}\left(t_{1}\right) c_{1}\left(t_{2}\right)^{2} \\
c_{n}\left(t_{1}+t_{2}\right)=c_{1}\left(t_{1}\right) c_{n}\left(t_{2}\right)+ \\
\sum_{k=2}^{n-1} c_{k}\left(t_{1}\right) \sum_{\bar{u}_{n} \in U_{n, k}} B_{\bar{u}_{n}} \prod_{j=1}^{n-k+1} c_{j}\left(t_{2}\right)^{u_{j}}+c_{n}\left(t_{1}\right) c_{1}\left(t_{2}\right)^{n}, n \in|3, s|
\end{array}\right.
$$

for $t_{1}, t_{2} \in G$.

\section{The regular one-parameter groups of formal power series}

We describe the regular one-parameter groups of FPS, i.e we give the general regular solution of the systems (13) and (14) for $s=\infty$. We recall the proofs of these results (see $[6,7,9]$ ) not only for the convenience of the reader. We prove these results in a more precise form, with some essential additional consequences, which allow us to give a simple proof of Theorem 2 .

In this section we assume that $c_{1}: \mathbb{K} \rightarrow \mathbb{K} \backslash\{0\}, c_{k}: \mathbb{K} \rightarrow \mathbb{K}$ for $k \geq 2$ are $C^{\infty}$-functions when $\mathbb{K}=\mathbb{R}$, or entire functions in the complex case. By the differentiation of each equation of (11) with respect to $t_{1}$, and putting $t_{1}=0$, jointly with the boundary condition $c_{1}(0)=1$ and $c_{n}(0)=0$ for each $n \geq p+2$ (cf. the translation equation (1)), we obtain the system of differential equations

$$
\left\{\begin{array}{l}
c_{1}^{\prime}(t)=c_{1}^{\prime}(0) c_{1}(t), \quad c_{1}(0)=1 \\
c_{2}^{\prime}(t)=c_{1}^{\prime}(0) c_{2}(t)+c_{2}^{\prime}(0) c_{1}(t)^{2}, \quad c_{2}(0)=0 \\
c_{n}^{\prime}(t)=c_{1}^{\prime}(0) c_{n}(t)+ \\
\sum_{k=2}^{n-1} c_{k}^{\prime}(0) \sum_{\bar{u}_{n} \in U_{n, k}} B_{\bar{u}_{n}} \prod_{j=1}^{n-k+1} c_{j}(t)^{u_{j}}+c_{n}^{\prime}(0) c_{1}(t)^{n}, c_{n}(0)=0, n \geq 3 .
\end{array}\right.
$$

Let us distinguish two cases:

c1) $c_{1}^{\prime}(0)=0$

c2) $c_{1}^{\prime}(0) \neq 0$.

In the first case, from the first differential equation of (15) we obtain $c_{1}=1$. Then, if for some integer $p \geq 0$ we have $c_{i}^{\prime}(0)=0$ for $i \in|2, p+1|$, and $c_{p+2}^{\prime}(0) \neq 0$, then from (15) we get $c_{i}=0$ for every $i \in|2, p+1|$, and $c_{p+2} \neq 0$. Hence from (15), for the sequence $\left(h_{n}\right)_{n \geq p+2}$, where $h_{k}:=c_{k}^{\prime}(0)$ for $k \geq p+2$, $h_{p+2} \neq 0$, we get

$$
\left\{\begin{array}{l}
c_{p+2}^{\prime}(t)=h_{p+2}, \quad c_{p+2}(0)=0, \\
c_{n}^{\prime}(t)=h_{n}+\sum_{k=p+2}^{n-p-1} h_{k} \sum_{\bar{u}_{n} \in U_{n, k}^{p}} B_{\bar{u}_{n}} \prod_{j=p+2}^{n-k+1} c_{j}(t)^{u_{j}}, c_{n}(0)=0, n \geq p+3 .
\end{array}\right.
$$

In the second case, for $\left(\lambda_{n}\right)_{n \geq 2}$, where $\lambda_{1}:=c_{1}^{\prime}(0)$ and $(k-1) \lambda_{1} \lambda_{k}:=c_{k}^{\prime}(0)$ for $k \geq 2$, we obtain 


$$
\left\{\begin{array}{l}
c_{1}^{\prime}(t)=\lambda_{1} c_{1}(t), \quad c_{1}(0)=1, \\
c_{2}^{\prime}(t)=\lambda_{1} c_{2}(t)+\lambda_{1} \lambda_{2} c_{1}(t)^{2}, \quad c_{2}(0)=0, \\
c_{n}^{\prime}(t)=\lambda_{1} c_{n}(t)+(n-1) \lambda_{1} \lambda_{n} c_{1}(t)^{n}+ \\
\quad \sum_{k=2}^{n-1}(k-1) \lambda_{1} \lambda_{k} \sum_{\bar{u}_{n} \in U_{n, k}} B_{\bar{u}_{n}} \prod_{j=1}^{n-k+1} c_{j}(t)^{u_{j}}, \quad c_{n}(0)=0, n \geq 3 .
\end{array}\right.
$$

Theorem 1. (cf. [6, Theorem 4] and [9, Theorem 6]) Fix an integer $p \geq 0$. There exist sequences of polynomials $\left(L_{n}^{p}\right)_{n \geq p+2}$ and $\left(P_{n}\right)_{n \geq 2}$ given by

$$
\left\{\begin{array}{l}
L_{p+2}^{p}(X)=0 ; \\
L_{n}^{p}\left(X ;\left(h_{l}\right)_{l \in|p+2, n-p-1|}\right)=\sum_{k=p+2}^{n-p-1} h_{k} \sum_{\bar{u}_{n} \in U_{n, k}^{p}} B_{\bar{u}_{n}} \\
\quad \int_{0}^{X}\left(\prod_{j=p+2}^{n-k+1}\left(h_{j} v+L_{j}^{p}\left(v ;\left(h_{l}\right)_{l \in|p+2, j-p-1|}\right)\right)^{u_{j}}\right) d v, n \geq p+3,
\end{array}\right.
$$

and by

$$
\left\{\begin{array}{c}
P_{2}(X)=0 ; \quad R_{2}\left(X ; \lambda_{2}\right)=\lambda_{2} X-\lambda_{2} \\
P_{n}\left(X ;\left(\lambda_{l}\right)_{l \in|2, n-1|}\right)=\sum_{k=2}^{n-1}(k-1) \lambda_{k} \sum_{\bar{u}_{n} \in U_{n, k}} B_{\bar{u}_{n}} \\
\quad \times \int_{1}^{X} v^{k-2} \prod_{j=2}^{n-k+1}\left(R_{j}\left(v ;\left(\lambda_{l}\right)_{l \in|2, j|}\right)\right)^{u_{j}} d v, \quad n \geq 3, \\
R_{n}\left(X ;\left(\lambda_{l}\right)_{l \in|2, n|}\right)=\lambda_{n}\left(X^{n-1}-1\right)+P_{n}\left(X ;\left(\lambda_{l}\right)_{l \in|2, n-1|}\right),
\end{array}\right.
$$

such that

(i) for every sequence $\left(h_{n}\right)_{n \geq p+2}$ there exists a unique solution of the system of differential equations (15) given by

$$
c_{n}(t)=h_{n} t+L_{n}^{p}\left(t ;\left(h_{k}\right)_{k \in|p+2, n-p-1|}\right), \quad t \in \mathbb{K}, n \geq p+2 ;
$$

(ii) for every sequence $\left(\lambda_{n}\right)_{n \geq 1}$ with $\lambda_{1} \neq 0$, there exists a unique solution of the system of differential equations (16) given by

$$
\begin{aligned}
& c_{1}(t)=e^{\lambda_{1} t}, \quad t \in \mathbb{K}, \\
& c_{n}(t)=\lambda_{n}\left(e^{n \lambda_{1} t}-e^{\lambda_{1} t}\right)+e^{\lambda_{1} t} P_{n}\left(e^{\lambda_{1} t} ;\left(\lambda_{k}\right)_{k \in|2, n-1|}\right), t \in \mathbb{K}, n \geq 2 .
\end{aligned}
$$

Proof. In the first case we have $c_{p+2}(t)=h_{p+2} t=h_{p+2} t+L_{p+2}^{p}(t)$ with $L_{p+2}^{p}=0$. Assume that for some $n \geq p+3$ there exist polynomials $L_{j}^{p}$ for $j \in|p+2, n-1|$ such that

$$
c_{j}(t)=h_{j} t+L_{j}^{p}\left(t ;\left(h_{l}\right)_{l \in|p+2, j-p-1|}\right), \quad t \in \mathbb{K}
$$


for every $j \in|2, n-1|$, and let us consider the differential equation

$$
\begin{aligned}
c_{n}^{\prime}(t) & =h_{n}+\sum_{k=p+2}^{n-p-1} h_{k} \sum_{\bar{u}_{n} \in U_{n, k}^{p}} B_{\bar{u}_{n}} \prod_{j=p+2}^{n-k+1} c_{j}(t)^{u_{j}} \\
& =h_{n}+\sum_{k=p+2}^{n-p-1} h_{k} \sum_{\bar{u}_{n} \in U_{n, k}^{p}} B_{\bar{u}_{n}} \prod_{j=p+2}^{n-k+1}\left(h_{j} t+L_{j}^{p}\left(t ;\left(h_{l}\right)_{l \in|p+2, j-p-1|}\right)\right)^{u_{j}}
\end{aligned}
$$

with the boundary condition $c_{n}(0)=0$. Since the right hand side of $(22)$ is a polynomial in $t$, this differential equation has a unique solution given by

$$
\begin{aligned}
c_{n}(t)= & h_{n} t \\
& +\sum_{k=p+2}^{n-p-1} h_{k} \sum_{\bar{u}_{n} \in U_{n, k}^{p}} B_{\bar{u}_{n}} \int_{0}^{t}\left(\prod_{j=p+2}^{n-k+1}\left(h_{j} v+L_{j}^{p}\left(v ;\left(h_{l}\right)_{l \in|p+2, j-p-1|}\right)\right)^{u_{j}}\right) d v .
\end{aligned}
$$

Hence

$$
\begin{aligned}
& L_{n}^{p}\left(X ;\left(h_{l}\right)_{l \in|p+2, n-p-1|}\right) \\
& \quad=\sum_{k=p+2}^{n-p-1} h_{k} \sum_{\bar{u}_{n} \in U_{n, k}^{p}} B_{\bar{u}_{n}} \int_{0}^{X}\left(\prod_{j=p+2}^{n-k+1}\left(h_{j} v+L_{j}^{p}\left(v ;\left(h_{l}\right)_{l \in|p+2, j-p-1|}\right)\right)^{u_{j}}\right) d v .
\end{aligned}
$$

Thus by (18) we can define the sequence of polynomials $\left(L_{n}^{p}\right)_{n \geq p+2}$ such that the solution of (16) is given by $(20)$.

Now, let $\lambda_{1}=c_{1}^{\prime}(0) \neq 0$. Then $c_{1}(t)=e^{\lambda_{1} t}$ and

$$
c_{2}(t)=\lambda_{2}\left(e^{2 \lambda_{1} t}-e^{\lambda_{1} t}\right)=\lambda_{2}\left(c_{1}^{2}(t)-c_{1}(t)\right)+c_{1}(t) P_{2}\left(c_{1}(t)\right)
$$

with $P_{2}(X)=0$. Assume that for some $n \in \mathbb{N}, n \geq 3$ there are polynomials $\left(P_{j}\right)_{j \in \mid 2, n-1} \mid$ such that

$$
c_{j}=\lambda_{j}\left(c_{1}^{j}-c_{1}\right)+c_{1} P_{j}\left(c_{1} ;\left(\lambda_{l}\right)_{l \in|2, j-1|}\right)=c_{1} R_{j}\left(c_{1} ;\left(\lambda_{l}\right)_{l \in|2, j|}\right)
$$

for every $j \in|2, n-1|$, and let us consider the differential equation

$$
c_{n}^{\prime}(t)=\lambda_{1} c_{n}(t)+(n-1) \lambda_{1} \lambda_{n} c_{1}(t)^{n}+\sum_{k=2}^{n-1}(k-1) \lambda_{1} \lambda_{k} \sum_{\bar{u}_{n} \in U_{n, k}} B_{\bar{u}_{n}} \prod_{j=1}^{n-k+1} c_{j}(t)^{u_{j}}
$$


with the boundary condition $c_{n}(0)=0$. We have

$$
\begin{aligned}
\prod_{j=1}^{n-k+1} c_{j}(t)^{u_{j}} & =c_{1}(t)^{u_{1}} \prod_{j=2}^{n-k+1}\left(c_{1}(t) R_{j}\left(c_{1}(t) ;\left(\lambda_{l}\right)_{l \in|2, j|}\right)\right)^{u_{j}} \\
& =c_{1}(t)^{\sum_{j=1}^{n-k+1} u_{j}} \prod_{j=2}^{n-k+1} R_{j}\left(c_{1}(t) ;\left(\lambda_{l}\right)_{l \in|2, j|}\right)^{u_{j}} \\
& =c_{1}(t)^{\sum_{j=1}^{n} u_{j}} \prod_{j=2}^{n-k+1} R_{j}\left(c_{1}(t) ;\left(\lambda_{l}\right)_{l \in|2, j|}\right)^{u_{j}} \\
& =c_{1}(t)^{k} \prod_{j=2}^{n-k+1} R_{j}\left(c_{1}(t) ;\left(\lambda_{l}\right)_{l \in|2, j|}\right)^{u_{j}} .
\end{aligned}
$$

Then, from (23), with $c_{1}(t)=e^{\lambda_{1} t}$ we get

$$
\begin{aligned}
c_{n}^{\prime}(t)= & \lambda_{1} c_{n}(t)+(n-1) \lambda_{1} \lambda_{n} e^{n \lambda_{1} t} \\
& +\sum_{k=2}^{n-1}(k-1) \lambda_{1} \lambda_{k} \sum_{\bar{u}_{n} \in U_{n, k}} B_{\bar{u}_{n}} e^{k \lambda_{1} t} \prod_{j=2}^{n-k+1} R_{j}\left(e^{\lambda_{1} t} ;\left(\lambda_{l}\right)_{l \in|2, j|}\right)^{u_{j}} .
\end{aligned}
$$

The linear differential equation (24) with the boundary condition $c_{n}(0)=0$ has exactly one solution (cf. [3, p. 104]) of the form

$$
\begin{aligned}
c_{n}(t)= & e^{\lambda_{1} t} \int_{0}^{t} e^{-\lambda_{1} v}(n-1) \lambda_{1} \lambda_{n} e^{(n-1) \lambda_{1} v} d v \\
& +e^{\lambda_{1} t} \int_{0}^{t} e^{-\lambda_{1} v} \sum_{k=2}^{n-1}(k-1) \lambda_{1} \lambda_{k} \sum_{\bar{u}_{n} \in U_{n, k}} B_{\bar{u}_{n}} e^{k \lambda_{1} v} \\
& \times \prod_{j=2}^{n-k+1} R_{j}\left(e^{\lambda_{1} v} ;\left(\lambda_{l}\right)_{l \in|2, j|}\right)^{u_{j}} d v \\
= & e^{\lambda_{1} t} \int_{1}^{e^{\lambda_{1} t}}(n-1) \lambda_{n} w^{n-2} d w \\
& +e^{\lambda_{1} t} \sum_{k=2}^{n-1}(k-1) \lambda_{k} \sum_{\bar{u}_{n} \in U_{n, k}} B_{\bar{u}_{n}} \int_{1}^{e^{\lambda_{1} t}} w^{k-2} \prod_{j=2}^{n-k+1} R_{j}\left(w ;\left(\lambda_{l}\right)_{l \in|2, j|}\right)^{u_{j}} d w .
\end{aligned}
$$

Thus

$$
c_{n}(t)=\lambda_{n}\left(e^{n \lambda_{1} t}-e^{\lambda_{1} t}\right)+e^{\lambda_{1} t} P_{n}\left(e^{\lambda_{1} t} ;\left(\lambda_{j}\right)_{j \in|2, n-1|}\right),
$$


where

$$
\begin{aligned}
& P_{n}\left(X ;\left(\lambda_{j}\right)_{j \in|2, n-1|}\right) \\
& \quad=\sum_{k=2}^{n-1}(k-1) \lambda_{k} \sum_{\bar{u}_{n} \in U_{n, k}} B_{\bar{u}_{n}} \int_{1}^{X} w^{k-2} \prod_{j=2}^{n-k+1}\left(R_{j}\left(w ;\left(\lambda_{l}\right)_{l \in|2, j|}\right)\right)^{u_{j}} d w .
\end{aligned}
$$

We have thus proved that there is a sequence of polynomials $\left(P_{n}\right)_{n \geq 2}$ defined by (19) such that the unique solution of (17) is given by (21).

Now we will show that the solutions $c_{k}$ of the systems of differential equations (16) and (17) satisfy the system of equations (11), which means that $F(t, X)=\sum_{k=1}^{\infty} c_{k}(t) X^{k}$ with $c_{k}$ given in Theorem 1 are the regular oneparameter groups of formal power series. In the following we use the standard notation $(s \in \mathbb{N}$ or $s=\infty)$. If $F(t, X)=\sum_{k=1}^{s} c_{k}(t) X^{k}$, then

$$
\frac{\partial F}{\partial X}(t, X):=\sum_{k=1}^{s} k c_{k}(t) X^{k-1},
$$

and, in the case when $G=\mathbb{K}$ and the coefficient functions $c_{k}$ are differentiable,

$$
\frac{\partial F}{\partial t}(t, X):=\sum_{k=1}^{s} c_{k}^{\prime}(t) X^{k} .
$$

For $G=\mathbb{K}$ the following theorem describes the general regular solution of the translation equation (1) in the ring of FPS. Theorem 2 may be derived from some results in $[12,13]$, but here we give the simple proof based on some ideas from [14].

Theorem 2. (i) If a family $(F(t, X))_{t \in \mathbb{K}}$ is a regular one-parameter group of $F P S$, then there exists a formal power series $H(X) \in \mathbb{K} \llbracket X \rrbracket$ such that

$$
\left\{\begin{array}{l}
\frac{\partial F}{\partial t}(t, X)=H(F(t, X)), \quad \text { for } t \in \mathbb{K}, \\
F(0, X)=X .
\end{array}\right.
$$

(ii) For each $H(X) \in \mathbb{K} \llbracket X \rrbracket$, ord $H \geq 1$, the family $(F(t, X))_{t \in \mathbb{K}}$ defined by (25) is a regular one-parameter group of formal power series.

(iii) The series $H$ is uniquely determined by $(F(t, X))_{t \in \mathbb{K}}$. It is given by the formula $H(X):=\frac{\partial F}{\partial t}(0, X)$, in particular, ord $H \geq 1$.

Proof. First let us assume that $(F(t, X))_{t \in \mathbb{K}}$ is a regular one-parameter group of FPS (i.e. $F(t, X)$ is a solution of the translation equation (1)). By differentiation of (1) with respect to $t_{1}$ and putting $t_{1}=0$ we get (25) with $H(X)=\frac{\partial F}{\partial t}(0, X)$, which is nothing else but (15).

Conversely, let us consider the system of differential equations (25) (which is equivalent to $(16)$ whenever $c_{1}^{\prime}(0)=0$, and to $(17)$ if $\left.c_{1}^{\prime}(0) \neq 0\right)$. We know 
that for a fixed $H(X) \in \mathbb{K} \llbracket X \rrbracket$ with ord $H \geq 1$, the system (25) always has a unique solution. We will show that it is given by (cf. also [12])

$$
F(t, X)=e^{t H(X) \frac{\partial}{\partial X}} X:=\sum_{k=0}^{\infty} \frac{t^{k}\left(H(X) \frac{\partial}{\partial X}\right)^{k}}{k !} X \quad \text { for } t \in \mathbb{K},
$$

where $\frac{\partial}{\partial X}$ denotes the operator of derivation with respect to $X$. Indeed, it is easy to see that the function $F(t, X)$ given by (26) is a regular solution of the translation equation (1) and $F(0, X)=X$. Thus, by differentiation of (1) with respect to $t_{1}$ and taking $t_{1}=0$ we get

$$
\frac{\partial F}{\partial t}(t, X)=\frac{\partial F}{\partial t}(0, F(t, X))
$$

Moreover, since

$$
\begin{aligned}
\frac{\partial F}{\partial t}(t, X) & =\frac{\partial}{\partial t}\left(e^{t H(X) \frac{\partial}{\partial X}} X\right) \\
& =\frac{\partial}{\partial t}\left(\sum_{k=0}^{\infty} \frac{\left(t^{k}\left(H(X) \frac{\partial}{\partial X}\right)^{k}\right.}{k !} X\right)=\sum_{k=1}^{\infty} \frac{t^{k-1}\left(H(X) \frac{\partial}{\partial X}\right)^{k}}{(k-1) !} X,
\end{aligned}
$$

$\frac{\partial F}{\partial t}(0, X)=H(X)$, and then, by $(27)$ we obtain (25). Thus every solution of the system of differential equations (25), with arbitrarily fixed $H(X) \in \mathbb{K} \llbracket X \rrbracket$ such that ord $H(X) \geq 1$, is a solution of the translation equation (1).

The proof of (iii) is obvious.

As a simple consequence of Theorem 1 and Theorem 2 we obtain

Theorem 3. There exist sequences of polynomials $\left(L_{n}^{p}\right)_{n \geq p+2}$ with some integer $p \geq 0$, and $\left(P_{n}\right)_{n \geq 2}$, given by (18) and (19), respectively, such that the coefficient functions of every regular nontrivial one-parameter group of FPS $F(t, X)=\sum_{k=1}^{\infty} c_{k}(t) X^{k}$ are given by $(20)$ provided $c_{1}^{\prime}(0)=0, c_{i}^{\prime}(0)=0$ for $i \in|2, p+1|, c_{p+1}^{\prime}(0) \neq 0$, and by $(21)$ if $c_{1}^{\prime}(0) \neq 0$, where $\left(h_{n}\right)_{n \geq p+2}$ and $\left(\lambda_{n}\right)_{n \geq 1}$ with $h_{p+2} \neq 0, \lambda_{1} \neq 0$ are arbitrary sequences of constants.

\section{Properties of the sequences $\left(L_{n}\right)$ and $\left(P_{n}\right)$}

Now we collect some properties of the polynomials $\left(L_{n}^{p}\right)$ and $\left(P_{n}\right)$ used for describing regular solutions of (11). First we quote without proof an interesting property of $\left(L_{n}^{p}\right)$ which can be derived from the construction of the regular one-parameter groups of FPS.

Fact 1. For every integer $p \geq 0$ and for every sequence of constants $\left(h_{n}\right)_{n \geq 2}$ with $h_{k}=0$ for $k \in|2, p+1|$ we have

$$
L_{n}^{0}\left(X ;\left(h_{l}\right)_{l \in|2, n-1|}\right)=L_{n}^{p}\left(X ;\left(h_{l}\right)_{l \in|p+2, n-p-1|}\right) \quad \text { for every } n \geq 2 \text {. }
$$


For every $n \geq p+2$ we denote

$$
\bar{L}_{n}^{p}\left(X ;\left(h_{l}\right)_{l \in|p+3, n-p-1|}\right):=L_{n}^{p}\left(X ;\left(h_{l}\right)_{l \in|p+2, n-p-1|}\right)
$$

with $h_{p+2}=1$. We prove now crucial properties of the polynomials $\bar{L}_{n}^{p}$ and $P_{n}$, which allow us to construct the general solution of systems (13) and (14). We begin with a well-known result from polynomial algebra.

Lemma 3. Let $A \subset \mathbb{K}$ be infinite. If $P(X, Y) \in \mathbb{K}[X, Y]$ and $P(a, b)=0$ for every $(a, b) \in A \times A$. Then $P(X, Y)=0$.

Now we prove

Lemma 4. For every $n \geq p+3$ the polynomials $\bar{L}_{n}^{p}$ given by (18) with $h_{p+2}=1$ satisfy, for an arbitrary sequence of constants $\left(h_{n}\right)_{n \geq p+3}$, the equalities

$$
\begin{aligned}
& h_{n}(X+Y)+\bar{L}_{n}^{p}\left(X+Y ;\left(h_{l}\right)_{l \in|p+3, n-p-1|}\right) \\
& =h_{n} X+\bar{L}_{n}^{p}\left(X ;\left(h_{l}\right)_{l \in|p+3, n-p-1|}\right)+h_{n} Y+\bar{L}_{n}^{p}\left(Y ;\left(h_{l}\right)_{l \in|p+3, n-p-1|}\right) \\
& \quad+\sum_{k=p+2}^{n-p-1} K_{k}^{p}\left(X ;\left(h_{l}\right)_{l \in|p+3, k|}\right) \sum_{\bar{u}_{n} \in U_{n, k}^{p}} B_{\bar{u}_{n}} \prod_{j=p+2}^{n-k+1} K_{j}^{p}\left(Y ;\left(h_{l}\right)_{l \in|p+3, j|}\right)^{u_{j}}
\end{aligned}
$$

and

$$
\begin{aligned}
(p+ & 2) X\left(h_{n} Y+\bar{L}_{n}^{p}\left(Y ;\left(h_{l}\right)_{l \in|p+3, n-p-1|}\right)\right) \\
& +n Y\left(h_{n} X+\bar{L}_{n}^{p}\left(X ;\left(h_{l}\right)_{l \in|p+3, n-p-1|}\right)\right) \\
& +\sum_{k=p+2}^{n-1} K_{k}^{p}\left(X ;\left(h_{l}\right)_{l \in|p+3, k|}\right) \sum_{\bar{u}_{r} \in \widehat{U}_{r, k}^{p}} B_{\bar{u}_{r}} \prod_{j=p+2}^{r-k+1} K_{j}^{p}\left(Y ;\left(h_{l}\right)_{l \in|p+3, j|}\right)^{u_{j}} \\
= & (p+2) Y\left(h_{n} X+\bar{L}_{n}^{p}\left(X ;\left(h_{l}\right)_{l \in|p+3, n-p-1|}\right)\right) \\
& +n X\left(h_{n} Y+\bar{L}_{n}^{p}\left(Y ;\left(h_{l}\right)_{l \in|p+3, n-p-1|}\right)\right) \\
& +\sum_{k=p+2}^{n-1} K_{k}^{p}\left(Y ;\left(h_{l}\right)_{l \in|p+3, k|}\right) \sum_{\bar{u}_{r} \in \widehat{U}_{r, k}^{p}} B_{\bar{u}_{r}} \prod_{j=p+2}^{r-k+1} K_{j}^{p}\left(X ;\left(h_{l}\right)_{l \in|p+3, j|}\right)^{u_{j}}
\end{aligned}
$$

in the ring $\mathbb{K}[X, Y]$, where $K_{p+2}^{p}(X)=X$, and

$$
K_{n}^{p}\left(X ;\left(h_{l}\right)_{l \in|p+3, n|}\right)=h_{n} X+\bar{L}_{n}^{p}\left(X ;\left(h_{l}\right)_{l \in|p+3, n-p-1|}\right) \text { for } n \geq p+3 .
$$

Proof. By Theorem 3, the family $F(t, X)=X+\sum_{j=p+2}^{\infty} \bar{c}_{j}(t) X^{j}$ with functions $\bar{c}_{j}: \mathbb{K} \rightarrow \mathbb{K}$ for $j \in|p+2, \infty|$ given by

$$
\begin{aligned}
& \bar{c}_{p+2}(t)=t, \quad t \in \mathbb{K}, \\
& \bar{c}_{n}(t)=h_{n} t+\bar{L}_{n}^{p}\left(t ;\left(h_{l}\right)_{l \in|p+3, n-p-1|}\right), t \in \mathbb{K}, n \in|p+3, \infty|,
\end{aligned}
$$


where $\left(h_{n}\right)_{n \geq p+3}$ is an arbitrary sequence of constants $\left(h_{p+2}=1\right)$, is a regular one-parameter group of FPS with $F(t, X) \equiv X \bmod X^{2}$. This means that the functions $\bar{c}_{n}$ for $n \geq p+2$ satisfy the system of equations (13). Then, for each $n \geq p+3$ we have

$$
\begin{aligned}
& h_{n}\left(t_{1}+t_{2}\right)+\bar{L}_{n}^{p}\left(t_{1}+t_{2} ;\left(h_{l}\right)_{l \in|p+3, n-p-1|}\right)=\bar{c}_{n}\left(t_{1}+t_{2}\right) \\
& =\bar{c}_{n}\left(t_{1}\right)+\bar{c}_{n}\left(t_{2}\right)+\sum_{k=p+2}^{n-p-1} \bar{c}_{k}\left(t_{1}\right) \sum_{\bar{u}_{n} \in U_{n, k}^{p}} B_{\bar{u}_{n}} \prod_{j=p+2}^{n-k+1} \bar{c}_{j}\left(t_{2}\right)^{u_{j}} \\
& =h_{n} t_{1}+\bar{L}_{n}^{p}\left(t_{1} ;\left(h_{l}\right)_{l \in|p+3, n-p-1|}\right)+h_{n} t_{2}+\bar{L}_{n}^{p}\left(t_{2},\left(h_{l}\right)_{l \in|p+3, n-p-1|}\right) \\
& \quad+\sum_{k=p+2}^{n-p-1} K_{k}^{p}\left(t_{1} ;\left(h_{l}\right)_{l \in|p+3, k|}\right) \sum_{\bar{u}_{n} \in U_{n, k}^{p}} B_{\bar{u}_{n}} \prod_{j=p+2}^{n-k+1} K_{j}^{p}\left(t_{2} ;\left(h_{l}\right)_{l \in|p+3, j|}\right)^{u_{j}}
\end{aligned}
$$

for every $t_{1}, t_{2} \in \mathbb{K}$. This jointly with Lemma 3 implies (29). Moreover, for every $n \geq p+3$ with $r=n+p+1$, on account of Corollary 4 , we have

$$
\begin{aligned}
\bar{c}_{r}\left(t_{1}+t_{2}\right)= & \bar{c}_{r}\left(t_{1}\right)+(p+2) t_{1}\left(h_{n} t_{2}+\bar{L}_{n}^{p}\left(t_{2} ;\left(h_{l}\right)_{l \in|p+3, n-p-1|}\right)\right) \\
& +\bar{c}_{r}\left(t_{2}\right)+n t_{2}\left(h_{n} t_{1}+\bar{L}_{n}^{p}\left(t_{1} ;\left(h_{l}\right)_{l \in|p+3, n-p-1|}\right)\right) \\
& +\sum_{k=p+2}^{n-1} K_{k}^{p}\left(t_{1} ;\left(h_{l}\right)_{l \in|p+3, k|}\right) \sum_{\bar{u}_{r} \in \widehat{U}_{r, k}^{p}} B_{\bar{u}_{r}} \prod_{j=p+2}^{r-k+1} K_{j}^{p}\left(t_{2} ;\left(h_{l}\right)_{l \in|p+3, j|}\right)^{u_{j}}
\end{aligned}
$$

for $t_{1}, t_{2} \in \mathbb{K}$. Since $(\mathbb{K},+)$ is an abelian group, we get by interchanging $t_{1}$ and $t_{2}$

$$
\begin{aligned}
(p+ & 2) t_{1}\left(h_{n} t_{2}+\bar{L}_{n}^{p}\left(t_{2} ;\left(h_{l}\right)_{l \in|p+3, n-p-1|}\right)\right)+ \\
& +n t_{2}\left(h_{n} t_{1}+\bar{L}_{n}^{p}\left(t_{1} ;\left(h_{l}\right)_{l \in|p+3, n-p-1|}\right)\right) \\
& +\sum_{k=p+2}^{n-1} K_{k}^{p}\left(t_{1} ;\left(h_{l}\right)_{l \in|p+3, k|}\right) \sum_{\bar{u}_{r} \in \widehat{U}_{r, k}^{p}} B_{\bar{u}_{r}} \prod_{j=p+2}^{r-k+1} K_{j}^{p}\left(t_{2} ;\left(h_{l}\right)_{l \in|p+3, j|}\right)^{u_{j}} \\
= & (p+2) t_{2}\left(h_{n} t_{1}+\bar{L}_{n}^{p}\left(t_{1} ;\left(h_{l}\right)_{l \in|p+3, n-p-1|}\right)\right) \\
& +n t_{1}\left(h_{n} t_{2}+\bar{L}_{n}^{p}\left(t_{2} ;\left(h_{l}\right)_{l \in|p+3, n-p-1|}\right)\right) \\
& +\sum_{k=p+2}^{n-1} K_{k}^{p}\left(t_{2} ;\left(h_{l}\right)_{l \in|p+3, k|}\right) \sum_{\bar{u}_{r} \in \widehat{U}_{r, k}^{p}} B_{\bar{u}_{r}} \prod_{j=p+2}^{r-k+1} K_{j}^{p}\left(t_{1} ;\left(h_{l}\right)_{l \in|p+3, j|}\right)^{u_{j}}
\end{aligned}
$$

which together with Lemma 3 gives (30). 
Lemma 5. Given an arbitrary sequence of constants $\left(\lambda_{n}\right)_{n \geq 2}$, the polynomials $P_{n}$ defined by (19) satisfy for every $n \geq 3$

$$
\begin{aligned}
\lambda_{n}( & \left.(X Y)^{n}-X Y\right)+X Y P_{n}\left(X Y ;\left(\lambda_{l}\right)_{l \in|2, n-1|}\right) \\
= & X\left(\lambda_{n}\left(Y^{n}-Y\right)+Y P_{n}\left(Y ;\left(\lambda_{l}\right)_{l \in|2, n-1|}\right)\right) \\
& +Y^{n}\left(\lambda_{n}\left(X^{n}-X\right)+X P_{n}\left(X ;\left(\lambda_{l}\right)_{l \in|2, n-1|}\right)\right) \\
& +\sum_{k=2}^{n-1} X R_{k}\left(X ;\left(\lambda_{l}\right)_{l \in|2, k|}\right) \sum_{\bar{u}_{n} \in U_{n, k}} B_{\bar{u}_{n}} Y^{k} \prod_{j=2}^{n-k+1}\left(R_{j}\left(Y ;\left(\lambda_{l}\right)_{l \in|2, j|}\right)\right)^{u_{j}},
\end{aligned}
$$

where $R_{1}(X)=X$ and

$$
R_{n}\left(X ;\left(\lambda_{l}\right)_{l \in|2, n|}\right)=\lambda_{n}\left(X^{n-1}-1\right)+P_{n}\left(X ;\left(\lambda_{l}\right)_{l \in|2, n-1|}\right) \text { for } n \in|2, \infty| \text {. }
$$

Proof. By Theorem 3 we have that the family $F(t, X)=e^{t} X+\sum_{k=1}^{\infty} \bar{c}_{k}(t) X^{k}$ with functions $\bar{c}_{k}: \mathbb{K} \rightarrow \mathbb{K}$ for $k \in|2, \infty|$ given by

$$
\bar{c}_{k}(t)=\lambda_{k}\left(e^{k t}-e^{t}\right)+e^{t} P_{k}\left(e^{t} ;\left(\lambda_{l}\right)_{l \in|2, k-1|}\right), \quad t \in \mathbb{K},
$$

and an arbitrary sequence $\left(\lambda_{n}\right)_{n \geq 2}$, is the regular one-parameter group of FPS with $F(t, X) \equiv e^{t} X \bmod X^{2}$. Hence the system of equations (14) with $\bar{c}_{1}(t)=e^{t}$ for $t \in \mathbb{K}$ is satisfied. Thus, for every $n \geq 2$ we have

$$
\begin{aligned}
\lambda_{n}\left(e^{n\left(t_{1}+t_{2}\right)}-e^{t_{1}+t_{2}}\right)+e^{t_{1}+t_{2}} P_{n}\left(e^{t_{1}+t_{2}} ; \lambda_{2}, \ldots, \lambda_{n-1}\right)=\bar{c}_{n}\left(t_{1}+t_{2}\right) \\
=\bar{c}_{1}\left(t_{1}\right) \bar{c}_{n}\left(t_{2}\right)+\bar{c}_{n}\left(t_{1}\right) \bar{c}_{1}\left(t_{2}\right)^{n}+\sum_{k=2}^{n-1} \bar{c}_{k}\left(t_{1}\right) \sum_{\bar{u}_{n} \in U_{n, k}} B_{\bar{u}_{n}} \prod_{j=1}^{n-k+1} \bar{c}_{j}\left(t_{2}\right)^{u_{j}} \\
=e^{t_{1}}\left(\lambda_{n}\left(e^{n t_{2}}-e^{t_{2}}\right)+e^{t_{2}} P_{n}\left(e^{t_{2}} ;\left(\lambda_{l}\right)_{l \in|2, n-1|}\right)\right) \\
\quad+e^{n t_{2}}\left(\lambda_{n}\left(e^{n t_{1}}-e^{t_{1}}\right)+e^{t_{1}} P_{n}\left(e^{t_{1}} ;\left(\lambda_{l}\right)_{l \in|2, n-1|}\right)\right) \\
\quad+\sum_{k=2}^{n-1} e^{t_{1}} R_{k}\left(e^{t_{1}} ; \lambda_{2}, \ldots, \lambda_{k}\right) \sum_{\bar{u}_{n} \in U_{n, k}} B_{\bar{u}_{n}} e^{k t_{2}} \prod_{j=2}^{n-k+1}\left(R_{j}\left(e^{t_{2}} ; \lambda_{2}, \ldots, \lambda_{j}\right)\right)^{u_{j}}
\end{aligned}
$$

for $t_{1}, t_{2} \in \mathbb{K}$. Since the function $\mathbb{K} \ni t \rightarrow e^{t} \in \mathbb{K}$ takes infinitely many values, Lemma 3 implies (31).

\section{One-parameter groups in cases 1 . and 3.}

Let $(G,+)$ be a commutative group, and let $s$ be a positive integer or $s=\infty$ (by $|\infty, \infty|$ we will mean $\emptyset$ ). We know that if $F^{[s]}(t, X)=X+\sum_{j=2}^{s} c_{j}(t) X^{j}$ is a solution of the translation equation with $c_{1}=1$, then we find $p \geq 0$ such that $p+2 \leq s, c_{k}=0$ for $k \in|2, p+1|$ and $c_{p+2}: G \rightarrow \mathbb{K}$ is a nonzero additive function. Then the functions $c_{k}: G \rightarrow \mathbb{K}$ for $k \in|p+2, s|$ satisfy the system of equations (13). We prove 
Theorem 4. Let the sequence of polynomials $\left(\bar{L}_{n}^{p}\right)_{n \geq p+2}$ be given by (28) and (18). For every one-parameter group $\Theta(t)(X)=X+\sum_{k=p+2}^{s} c_{k}(t) X^{k}$ of FPS with $c_{p+2} \neq 0$, there exist a nonzero additive function $a: G \rightarrow \mathbb{K}$, a sequence of additive functions $\left(a_{n}\right)_{n \in|s-p, s|}, a_{n}: G \rightarrow \mathbb{K}$, and a sequence of constants $\left(h_{n}\right)_{n \in|p+3, s-p-1|}$ such that the coefficient functions $\left(c_{k}\right)_{k \in|p+2, s|}$ are given by $c_{p+2}(t)=a(t), t \in G$

$c_{n}(t)=h_{n} a(t)+\bar{L}_{n}^{p}\left(a(t) ;\left(h_{j}\right)_{j \in|p+3, n-p-1|}\right), t \in G, n \in|p+3, s-p-1|$,

$\left.c_{n}(t)=a_{n}(t)+\bar{L}_{n}^{p}\left(a(t) ;\left(h_{j}\right)_{j \in|p+3, n-p-1|}\right)\right), t \in G, n \in|s-p, s|$.

Conversely, for an arbitrary additive function $a: G \rightarrow \mathbb{K}$, for each sequence $\left(h_{n}\right)_{n \in|p+3, s-p-1|}$ and for every sequence of additive functions $\left(a_{n}\right)_{n \in|s-p, s|}, a_{n}: G \rightarrow \mathbb{K}$, a function $\Theta(t)(X)=X+\sum_{k=p+2}^{s} c_{k}(t) X^{k}$ defined by (32) is a one-parameter group of FPS.

Proof. At first we prove that the functions $c_{n}$ for $n \in|p+2, s|$ given by (32) satisfy the system of equations (13). It is easy to see that the function $c_{p+2}$ satisfy the first equation of the system (13). So let us fix $n \in|p+3, s-p-1|$. Then, using (29), for arbitrary $t_{1}, t_{2} \in G$ we get

$$
\begin{aligned}
c_{n}\left(t_{1}+t_{2}\right)= & h_{n} a\left(t_{1}+t_{2}\right)+\bar{L}_{n}^{p}\left(a\left(t_{1}+t_{2}\right) ;\left(h_{l}\right)_{l \in|p+3, n-p-1|}\right) \\
= & h_{n}\left(a\left(t_{1}\right)+a\left(t_{2}\right)\right)+\bar{L}_{n}^{p}\left(a\left(t_{1}\right)+a\left(t_{2}\right) ;\left(h_{l}\right)_{l \in|p+3, n-p-1|}\right) \\
= & h_{n} a\left(t_{1}\right)+\bar{L}_{n}^{p}\left(a\left(t_{1}\right) ;\left(h_{l}\right)_{l \in|p+3, n-p-1|}\right) \\
& +h_{n} a\left(t_{2}\right)+\bar{L}_{n}^{p}\left(a\left(t_{2}\right) ;\left(h_{l}\right)_{l \in|p+3, n-p-1|}\right) \\
& \quad+\sum_{k=p+2}^{n-p-1} K_{k}^{p}\left(a\left(t_{1}\right) ;\left(h_{l}\right)_{l \in|p+3, k|}\right) \sum_{\bar{u}_{n} \in U_{n, k}^{p}} B_{\bar{u}_{n}} \\
& \times \prod_{j=p+2}^{n-k+1} K_{j}^{p}\left(a\left(t_{2}\right) ;\left(h_{l}\right)_{l \in|p+3, j|}\right)^{u_{j}} \\
= & c_{n}\left(t_{1}\right)+c_{n}\left(t_{2}\right)+\sum_{k=p+2}^{n-p-1} c_{k}\left(t_{1}\right) \sum_{\bar{u}_{n} \in U_{n, k}^{p}} B_{\bar{u}_{n}} \prod_{j=p+2}^{n-k+1} c_{j}\left(t_{2}\right)^{u_{j}} .
\end{aligned}
$$

Finally, fix $n \in|s-p, s|$. Then using (29) with $h_{n}=0$ we obtain

$$
\begin{aligned}
& \bar{L}_{n}^{p}\left(a\left(t_{1}+t_{2}\right) ;\left(h_{l}\right)_{l \in|p+3, n-p-1|}\right) \\
& =\bar{L}_{n}^{p}\left(a\left(t_{1}\right)+a\left(t_{2}\right) ;\left(h_{l}\right)_{l \in|p+3, n-p-1|}\right) \\
& =\bar{L}_{n}^{p}\left(a\left(t_{1}\right) ;\left(h_{l}\right)_{l \in|p+3, n-p-1|}\right)+\bar{L}_{n}^{p}\left(a\left(t_{2}\right) ;\left(h_{l}\right)_{l \in|p+3, n-p-1|}\right) \\
& \quad+\sum_{k=p+2}^{n-p-1} K_{k}^{p}\left(a\left(t_{1}\right) ;\left(h_{l}\right)_{l \in|p+3, k|}\right) \sum_{\bar{u}_{n} \in U_{n, k}^{p}} B_{\bar{u}_{n}} \\
& \quad \times \prod_{j=p+2}^{n+1} K_{j}^{p}\left(a\left(t_{2}\right) ;\left(h_{l}\right)_{l \in|p+3, j|}\right)^{u_{j}}
\end{aligned}
$$


Consequently, with an arbitrary additive function $a_{n}: G \rightarrow \mathbb{K}$ we get

$$
\begin{aligned}
c_{n}\left(t_{1}+t_{2}\right)= & a_{n}\left(t_{1}+t_{2}\right)+\bar{L}_{n}^{p}\left(a\left(t_{1}+t_{2}\right) ;\left(h_{l}\right)_{l \in|p+3, n-p-1|}\right) \\
= & a_{n}\left(t_{1}\right)+a_{n}\left(t_{2}\right)+\bar{L}_{n}^{p}\left(a\left(t_{1}\right)+a\left(t_{2}\right) ;\left(h_{l}\right)_{l \in|p+3, n-p-1|}\right) \\
= & a_{n}\left(t_{1}\right)+\bar{L}_{n}^{p}\left(a\left(t_{1}\right) ;\left(h_{l}\right)_{l \in|p+3, n-p-1|}\right) \\
& +a_{n}\left(t_{2}\right)+\bar{L}_{n}^{p}\left(a\left(t_{2}\right) ;\left(h_{l}\right)_{l \in|p+3, n-p-1|}\right) \\
& +\sum_{k=p+2}^{n-p-1} K_{k}^{p}\left(a\left(t_{1}\right) ;\left(h_{l}\right)_{l \in|p+3, k|}\right) \sum_{\bar{u}_{n} \in U_{n, k}^{p}} B_{\bar{u}_{n}} \\
& \quad \times \prod_{j=p+2}^{n-k+1} K_{j}^{p}\left(a\left(t_{2}\right) ;\left(h_{l}\right)_{l \in|p+3, j|}\right)^{u_{j}} \\
= & c_{n}\left(t_{1}\right)+c_{n}\left(t_{2}\right)+\sum_{k=p+2}^{n-p-1} c_{k}\left(t_{1}\right) \sum_{\bar{u}_{n} \in U_{n, k}^{p}} B_{\bar{u}_{n}} \prod_{j=p+2}^{n-k+1} c_{j}\left(t_{2}\right)^{u_{j}},
\end{aligned}
$$

which proves that the functions $c_{n}, n \in|p+2, s|$, defined by (32) satisfy the system of equations (13) with arbitrary constants $\left(h_{n}\right)_{n \in|p+3, s-p-1|}$ and arbitrary additive functions $a_{n}: G \rightarrow \mathbb{K}$ for $n \in|s-p, s|$.

Now we are going to show that functions defined by (32) are the only solutions of (13). Here we use an approach much simpler than the one applied in $[6,7]$. Note that from (13) jointly with $c_{p+2} \neq 0$ it follows that $c_{p+2}$ is a nonzero additive function. So let $0 \neq c_{p+2}=a: G \rightarrow \mathbb{K}$ be an additive function.

Put $h_{p+2}=1$ and $\bar{L}_{p+2}^{p}(X)=0$. Assume that for some $n \in|p+3, s-p-1|$

$$
c_{j}(t)=h_{j} a(t)+\bar{L}_{j}^{p}\left(a(t) ;\left(h_{l}\right)_{l \in|p+3, j-p-1|}\right), t \in G, j \in|p+2, n-1| .
$$

Let us consider the equation with a function $c_{n}$ on the left hand side of it, that is

$$
\begin{aligned}
c_{n}\left(t_{1}+t_{2}\right)= & c_{n}\left(t_{1}\right)+c_{n}\left(t_{2}\right) \\
& +\sum_{k=p+2}^{n-p-1} K_{k}^{p}\left(a\left(t_{1}\right) ;\left(h_{l}\right)_{l \in|p+3, k|}\right) \sum_{\bar{u}_{n} \in U_{n, k}^{p}} B_{\bar{u}_{n}} \\
& \times \prod_{j=p+2}^{n-k+1} K_{j}^{p}\left(a\left(t_{2}\right) ;\left(h_{l}\right)_{l \in|p+3, j|}\right)^{u_{j}}
\end{aligned}
$$

for $t_{1}, t_{2} \in \mathbb{K}$, which is, in fact, an inhomogeneous Cauchy equation for $c_{n}$ provided $a$ and $\left(h_{l}\right)_{l \in|p+3, n-p-1|}$ are given. Hence the general solution of (33) is a sum of a particular solution of this equation and an additive function. Moreover, for every $t_{1}, t_{2} \in G$, on account of (29) with $h_{n}=0$, we have 


$$
\begin{aligned}
& \bar{L}_{n}^{p}\left(a\left(t_{1}+t_{2}\right) ;\left(h_{l}\right)_{l \in|p+3, n-p-1|}\right)=\bar{L}_{n}^{p}\left(a\left(t_{1}\right)+a\left(t_{2}\right) ;\left(h_{l}\right)_{l \in|p+3, n-p-1|}\right) \\
& =\bar{L}_{n}^{p}\left(a\left(t_{1}\right) ;\left(h_{l}\right)_{l \in|p+3, n-p-1|}\right)+\bar{L}_{n}^{p}\left(a\left(t^{\prime}\right) ;\left(h_{l}\right)_{l \in|p+3, n-p-1|}\right) \\
& \quad+\sum_{k=p+2}^{n-p-1} K_{k}^{p}\left(a\left(t_{1}\right) ;\left(h_{l}\right)_{l \in|p+3, k|}\right) \sum_{\bar{u}_{n} \in U_{n, k}^{p}} B_{\bar{u}_{n}} \prod_{j=p+2}^{n-k+1} K_{j}^{p}\left(a\left(t_{2}\right) ;\left(h_{l}\right)_{l \in|p+3, j|}\right)^{u_{j}}
\end{aligned}
$$

which proves that $\bar{L}_{n}^{p}\left(a(t) ;\left(h_{l}\right)_{l \in|p+3, n-p-1|}\right)$ is a solution of (33). Thus every solution of (33) must be of the form

$$
c_{n}(t)=a_{n}(t)+\bar{L}_{n}^{p}\left(a(t) ;\left(h_{l}\right)_{l \in|p+3, n-p-1|}\right) \quad t \in G
$$

with an additive function $a_{n}: \mathbb{K} \rightarrow \mathbb{K}$. We will show that $a_{n}=h_{n} a$ for $n \in|p+3, s-p-1|$. Let us consider the equation of the system (13) with the function $c_{n+p+1}$ on the left hand side of it. By (34) and Corollary 4 with $r=n+p+1$ it can be written as

$$
\begin{aligned}
c_{r}\left(t_{1}\right. & \left.+t_{2}\right) \\
= & c_{r}\left(t_{1}\right)+(p+2) a\left(t_{1}\right)\left(a_{n}\left(t_{2}\right)+\bar{L}_{n}^{p}\left(a\left(t_{2}\right) ;\left(h_{l}\right)_{l \in|p+3, n-p-1|}\right)\right) \\
& +c_{r}\left(t_{2}\right)+n a\left(t_{2}\right)\left(a_{n}\left(t_{1}\right)+\bar{L}_{n}^{p}\left(a\left(t_{1}\right) ;\left(h_{l}\right)_{l \in|p+3, n-p-1|}\right)\right) \\
& +\sum_{k=p+2}^{n-p-1} K_{k}^{p}\left(a\left(t_{1}\right) ;\left(h_{l}\right)_{l \in|p+3, k|}\right) \sum_{\bar{u}_{r} \in \widehat{U}_{r, k}^{p}} B_{\bar{u}_{r}} \prod_{j=p+2}^{r-k+1} K_{j}^{p}\left(a\left(t_{2}\right) ;\left(h_{l}\right)_{l \in|p+3, j|}\right)^{u_{j}}
\end{aligned}
$$

for $t_{1}, t_{2} \in \mathbb{K}$. Since $(G,+)$ is an abelian group, by interchanging $t_{1}$ and $t_{2}$ in the above equality we get

$$
\begin{aligned}
& (p+2) a\left(t_{1}\right)\left(a_{n}\left(t_{2}\right)+\bar{L}_{n}^{p}\left(a\left(t_{2}\right) ;\left(h_{l}\right)_{l \in|p+3, n-p-1|}\right)\right) \\
& +n a\left(t_{2}\right)\left(a_{n}\left(t_{1}\right)+\bar{L}_{n}^{p}\left(a\left(t_{1}\right) ;\left(h_{l}\right)_{l \in|p+3, n-p-1|}\right)\right) \\
& +\sum_{k=p+2}^{n-1} K_{k}^{p}\left(a\left(t_{1}\right) ;\left(h_{l}\right)_{l \in|p+3, k|}\right) \sum_{\bar{u}_{r} \in \widehat{U}_{r, k}^{p}} B_{\bar{u}_{r}} \prod_{j=p+2}^{r-k+1} K_{j}^{p}\left(a\left(t_{2}\right) ;\left(h_{l}\right)_{l \in|p+3, j|}\right)^{u_{j}} \\
& =c_{r}\left(t_{1}+t_{2}\right)=c_{r}\left(t_{2}+t_{1}\right) \\
& =(p+2) a\left(t_{2}\right)\left(a_{n}\left(t_{1}\right)+\bar{L}_{n}^{p}\left(a\left(t_{1}\right) ;\left(h_{l}\right)_{l \in|p+3, n-p-1|}\right)\right) \\
& +n a\left(t_{1}\right)\left(a_{n}\left(t_{2}\right)+\bar{L}_{n}^{p}\left(a\left(t_{2}\right) ;\left(h_{l}\right)_{l \in|p+3, n-p-1|}\right)\right) \\
& +\sum_{k=p+2}^{n-1} K_{k}^{p}\left(a\left(t_{2}\right) ;\left(h_{l}\right)_{l \in|p+3, k|}\right) \sum_{\bar{u}_{r} \in \widehat{U}_{r, k}^{p}} B_{\bar{u}_{r}} \prod_{j=p+2}^{r-k+1} K_{j}^{p}\left(a\left(t_{1}\right) ;\left(h_{l}\right)_{l \in|p+3, j|}\right)^{u_{j}} .
\end{aligned}
$$


From the last relation, using (30) with $h_{n}=0$ we get

$$
(p+2) a\left(t_{1}\right) a_{n}\left(t_{2}\right)+n a\left(t_{2}\right) a_{n}\left(t_{1}\right)=(p+2) a\left(t_{2}\right) a_{n}\left(t_{1}\right)+n a\left(t_{1}\right) a_{n}\left(t_{2}\right)
$$

for $t_{1}, t_{2} \in G$. Since $n \geq p+3$, we have

$$
a\left(t_{1}\right) a_{n}\left(t_{2}\right)=a\left(t_{2}\right) a_{n}\left(t_{1}\right) \text { for } t_{1}, t_{2} \in G .
$$

Fix $t_{1} \in G$ with $a\left(t_{1}\right)=c_{p+2}\left(t_{1}\right) \neq 0$. Then with $h_{n}:=\frac{a_{n}\left(t_{1}\right)}{a\left(t_{1}\right)} \in \mathbb{K}$ we get $a_{n}=h_{n} a$, and consequently, from (14) we obtain

$$
c_{n}(t)=h_{n} a(t)+\bar{L}_{n}^{p}\left(a(t) ;\left(h_{l}\right)_{l \in|p+3, n-p-1|}\right) \quad \text { for } t \in G .
$$

Now, fix $n \in|s-p, s|$ (clearly then $s<\infty$ ) and assume that

$$
\begin{aligned}
& c_{p+2}(t)=a(t), \\
& c_{j}(t)=h_{j} a(t)+\bar{L}_{j}^{p}\left(a(t) ;\left(h_{l}\right)_{l \in|p+3, n-p-1|}\right), \quad j \in|p+3, s-p-1|, \\
& c_{j}(t)=a_{j}(t)+\bar{L}_{j}^{p}\left(a(t) ;\left(h_{l}\right)_{l \in|p+3, n-p-1|}\right), \quad j \in|s-p, n-1| .
\end{aligned}
$$

Let us consider once more Eq. (33). The same way as before we show that then $c_{n}$ must be of the form (34). Since in this case $n+p+1>s$, this finishes the proof.

Assume now that $s$ is a positive integer or $s=\infty$ and $F^{[s]}(t, X)=$ $\sum_{i=1}^{s} c_{i}(t) X^{i}$ is a solution of the translation equation ((1) if $s=\infty$ and (2) in the case when $s$ is finite) with $c_{1} \neq 1$. Then the functions $c_{i}$ satisfy the system of equations (14) and $c_{1}$ is a generalized exponential function. We are going to consider firstly the already mentioned subcase where $c_{1}$ takes infinitely many values. In what follows, we will need

Lemma 6. Assume that $(G,+)$ is a commutative group, $k \geq 2$ is a positive integer and let $f: G \rightarrow \mathbb{K} \backslash\{0\}$ be an exponential function such that $f\left(y_{0}\right)^{k}-$ $f\left(y_{0}\right) \neq 0$ for some $y_{0} \in G$. If a function $g: G \rightarrow \mathbb{K}$ satisfies the equation

$$
g(x+y)=f(x) g(y)+f(y)^{k} g(x) \text { for } x, y \in G,
$$

where $k \geq 2$ is an integer, then there exists a constant $p \in \mathbb{K}$ such that

$$
g(x)=p\left(f(x)^{k}-f(x)\right) \text { for } x \in G .
$$

Proof. From the symmetry of the left hand side of (35), we get for every $x, y \in G$

$$
f(x) g(y)+f(y)^{k} g(x)=g(x+y)=g(y+x)=f(y) g(x)+f(x)^{k} g(y),
$$

or, equivalently, $g(x)\left(f(y)^{k}-f(y)\right)=g(y)\left(f(x)^{k}-f(x)\right)$. Put $y=y_{0}$. Then, with $p=\frac{g\left(y_{0}\right)}{f\left(y_{0}\right)^{k}-f\left(y_{0}\right)}$ we obtain (36). 
Theorem 5. Let $(G,+)$ be a commutative group which admits a generalized exponential function from $G$ into $\mathbb{K} \backslash\{0\}$ with infinite image. There exists a sequence of polynomials $\left(P_{n}\right)_{n \geq 2}$ defined by (19) such that for every oneparameter group $\Theta(t)(X)=\sum_{k=1}^{s} c_{k}(t) X^{k}$ of FPS with a generalized exponential function $c_{1}$ taking infinitely many values, there exists a sequence of constants $\left(\lambda_{n}\right)_{n \in|2, s|}$ such that for every $n \in|2, s|$

$$
c_{n}(t)=\lambda_{n}\left(c_{1}(t)^{n}-c_{1}(t)\right)+c_{1}(t) P_{n}\left(c_{1}(t) ;\left(\lambda_{l}\right)_{l \in|2, n-1|}\right) \quad \text { for } t \in G .
$$

Conversely, for each exponential function $c_{1}$ and for each sequence $\left(\lambda_{n}\right)_{n \in|2, s|}$, the function $\Theta(t)(X)=\sum_{k=1}^{s} c_{k}(t) X^{k}$ is a one-parameter group of FPS.

Proof. The proof of Theorem 5 is based in the same ideas as the proof of Theorem 4 and it is easier than the one given in [9]. We show first that with an arbitrary exponential function $c_{1}$ the functions $\left(c_{n}\right)_{n \in|2, s|}$ defined by (37) satisfy the system of equations (14). Indeed, fix $n \geq 2$, a sequence $\left(\lambda_{n}\right)_{n \in|2, s|}$ and $t_{1}, t_{2} \in G$. Using Lemma 5 we get

$$
\begin{aligned}
c_{n}( & \left.t_{1}+t_{2}\right) \\
= & \lambda_{n}\left(c_{1}\left(t_{1}+t_{2}\right)^{n}-c_{1}\left(t_{1}+t_{2}\right)\right)+c_{1}\left(t_{1}+t_{2}\right) P_{n}\left(c_{1}\left(t_{1}+t_{2}\right) ;\left(\lambda_{l}\right)_{l \in|2, n-1|}\right) \\
= & \lambda_{n}\left(\left(c_{1}\left(t_{1}\right) c_{1}\left(t_{2}\right)\right)^{n}-c_{1}\left(t_{1}\right) c_{1}\left(t_{2}\right)\right) \\
& +c_{1}\left(t_{1}\right) c_{1}\left(t_{2}\right) P_{n}\left(c_{1}\left(t_{1}\right) c_{1}\left(t_{2}\right) ;\left(\lambda_{l}\right)_{l \in|2, n-1|}\right) \\
= & c_{1}\left(t_{1}\right)\left(\lambda_{n}\left(c_{1}\left(t_{2}\right)^{n}-c_{1}\left(t_{2}\right)\right)+c_{1}\left(t_{2}\right) P_{n}\left(c_{1}\left(t_{2}\right) ;\left(\lambda_{l}\right)_{l \in|2, n-1|}\right)\right) \\
& +c_{1}\left(t_{2}\right)^{n}\left(\lambda_{n}\left(c_{1}\left(t_{1}\right)^{n}-c_{1}\left(t_{1}\right)\right)+c_{1}\left(t_{1}\right) P_{n}\left(c_{1}\left(t_{1}\right) ;\left(\lambda_{l}\right)_{l \in|2, n-1|}\right)\right) \\
& +\sum_{k=2}^{n-1} c_{1}\left(t_{1}\right) R_{k}\left(c_{1}\left(t_{1}\right) ;\left(\lambda_{l}\right)_{l \in|2, k|}\right) \sum_{\bar{u}_{n} \in U_{n, k}} B_{\bar{u}_{n}} c_{1}\left(t_{2}\right)^{k} \\
& \quad \times \prod_{j=2}^{n-k+1} R_{j}\left(c_{1}\left(t_{2}\right) ;\left(\lambda_{l}\right)_{l \in|2, j|}\right)^{u_{j}}{ }_{n-1}^{n-1} c_{k}\left(t_{1}\right) \sum_{\bar{u}_{n} \in U_{n, k}} \prod_{j=1}^{n-k+1} c_{j}\left(t_{2}\right)^{u_{j} .} .
\end{aligned}
$$

Now we are going to prove that the functions defined by (37) are the unique ones satisfying the system of equations (14). From (14) it follows that $c_{1}$ is an exponential function and assume that it takes infinitely many values. Then, by Lemma 6 ,

$$
c_{2}(t)=\lambda_{2}\left(c_{1}(t)^{2}-c_{1}(t)\right)=\lambda_{2}\left(c_{1}(t)^{2}-c_{1}(t)\right)+c_{1}(t) P_{2}\left(c_{1}(t)\right) \text { for } t \in G,
$$

where $P_{2}(X)=0$. Assume now that for some $n \in|3, s|$ we have $c_{j}(t)=\lambda_{n}\left(c_{1}(t)^{j}-c_{1}(t)\right)+c_{1}(t) P_{j}\left(c_{1}(t) ;\left(\lambda_{l}\right)_{l \in|2, j-1|}\right), \quad t \in G, j \in|2, n-1|$, 
and consider the equation of the system (14) with the function $c_{n}$ on the left hand side, i.e.

$$
\begin{aligned}
c_{n}\left(t_{1}+t_{2}\right)= & c_{1}\left(t_{1}\right) c_{n}\left(t_{2}\right)+c_{n}\left(t_{1}\right) c_{1}\left(t_{2}\right)^{n} \\
& +\sum_{k=2}^{n-1} c_{1}\left(t_{1}\right) R_{k}\left(c_{1}\left(t_{1}\right) ;\left(\lambda_{l}\right)_{l \in|2, k|}\right) \sum_{\bar{u}_{n} \in U_{n, k}} B_{\bar{u}_{n}} \\
& \times c_{1}\left(t_{2}\right)^{k} \prod_{j=1}^{n-k+1} R_{j}\left(c_{1}\left(t_{2}\right) ;\left(\lambda_{l}\right)_{l \in|2, j|}\right)^{u_{j}}
\end{aligned}
$$

for $t_{1}, t_{2} \in G$. Note that if the functions $c_{n}^{1}, c_{n}^{2}: G \rightarrow \mathbb{K}$ satisfy (38), then the difference $f=c_{n}^{2}-c_{n}^{1}$ satisfies the equation

$$
f\left(t_{1}+t_{2}\right)=c_{1}\left(t_{1}\right) f\left(t_{2}\right)+c_{1}\left(t_{2}\right)^{n} f\left(t_{1}\right) \text { for } t_{1}, t 2 \in G .
$$

Thus, on account of Lemma 6 , every solution of (38) is a sum of a particular solution of (38) and a function $f(t)=\lambda_{n}\left(c_{1}(t)^{n}-c_{1}(t)\right)$. Moreover, from Lemma 5 with $\lambda_{n}=0$ we obtain

$$
\begin{aligned}
& c_{1}\left(t_{1}+t_{2}\right) P_{n}\left(c_{1}\left(t_{1}+t_{2}\right) ;\left(\lambda_{l}\right)_{l \in|2, n-1|}\right) \\
& =c_{1}\left(t_{1}\right) c_{1}\left(t_{2}\right) P_{n}\left(c_{1}\left(t_{1}\right) c_{1}\left(t_{2}\right) ;\left(\lambda_{l}\right)_{l \in|2, n-1|}\right) \\
& =c_{1}\left(t_{1}\right) c_{1}\left(t_{2}\right) P_{n}\left(c_{1}\left(t_{2}\right) ;\left(\lambda_{l}\right)_{l \in|2, n-1|}\right) \\
& +c_{1}\left(t_{2}\right)^{n} c_{1}\left(t_{1}\right) P_{n}\left(c_{1}\left(t_{1}\right) ;\left(\lambda_{l}\right)_{l \in|2, n-1|}\right) \\
& \quad+\sum_{k=2}^{n-1} c_{1}\left(t_{1}\right) R_{k}\left(c_{1}\left(t_{1}\right) ;\left(\lambda_{l}\right)_{l \in|2, k|}\right) \sum_{\bar{u}_{n} \in U_{n, k}} B_{\bar{u}_{n}} \\
& \quad \times c_{1}\left(t_{2}\right)^{k} \prod_{j=1}^{n-k+1} R_{j}\left(c_{1}\left(t_{2}\right) ;\left(\lambda_{l}\right)_{l \in|2, j|}\right)^{u_{j}} .
\end{aligned}
$$

This means that $c_{1}(t) P_{n}\left(c_{1}(t) ;\left(\lambda_{l}\right)_{l \in|2, n-1|}\right)$ is a solution of (38). Thus

$$
c_{n}(t)=\lambda_{n}\left(c_{1}(t)^{n}-c_{1}(t)\right)+c_{1}(t) P_{n}\left(c_{1}(t) ;\left(\lambda_{l}\right)_{l \in|2, n-1|}\right), \quad t \in G,
$$

which completes the proof.

\section{One-parameter groups in case 2.}

We describe the one-parameter groups $\Theta_{s}: G \rightarrow \Gamma^{s}, \Theta_{s}(t)(X)=$ $\sum_{k=1}^{s} c_{k}(t) X^{k}$ where $s$ is a positive integer or $s=\infty$ and $c_{1}: G \rightarrow \mathbb{K} \backslash\{0\}$ $c_{1} \neq 1$ is a generalized exponential function taking finitely many values and $c_{k}: G \rightarrow \mathbb{K}$ for $k \in|2, s|$. The proposed construction depends strictly on the form of an exponential function $c_{1}$. Note, that if $(G,+)$ is a commutative group and if $c_{1}: G \rightarrow \mathbb{K} \backslash\{0\}$ is a generalized exponential function such that $\operatorname{im} c_{1}=E_{m}$ with an integer $m \geq 2$, then for a fixed $t_{0} \in G$ such that $c_{1}\left(t_{0}\right)$ is a primitive root of 1 of order $m$, we have then $G / \operatorname{ker} c_{1}=\left\{l t_{0}+\operatorname{ker} c_{1}: 0 \leq\right.$ 
$l \leq m-1\}, m t_{0} \in \operatorname{ker} c_{1}$ and every $t \in G$ may be uniquely written as $l t_{0}+t^{\prime}$ with some $0 \leq l \leq m-1$ and $t^{\prime} \in \operatorname{ker} c_{1}$.

We prove

Proposition 1. Let $s$ be a positive integer or $s=\infty$. If $\Theta_{s}: G \rightarrow \Gamma^{s}$, $\Theta_{s}(t)(X)=\sum_{k=1}^{s} c_{k}(t) X^{k}$ is a one-parameter group of FPS, then $\left.\Theta_{s}\right|_{\operatorname{ker} c_{1}}=$ $\bar{\Theta}_{s}: \operatorname{ker} c_{1} \rightarrow \Gamma_{1}^{s}, \bar{\Theta}_{s}(t)(X)=X+\sum_{k=2}^{s} \bar{c}_{k}(t) X^{k}$ is a one-parameter group of FPS, and there exists $t_{0} \in G$ and a FPS $P(X)=\Theta_{s}\left(t_{0}\right)(X)=\sum_{k=1}^{s} d_{k} X^{k}$ $\left(d_{1}=c_{1}\left(t_{0}\right)\right.$ is a primitive root of 1 of order $\left.m\right)$ such that

$$
\begin{aligned}
\left(P \circ \bar{\Theta}_{s}(t)\right)(X) & =\left(\bar{\Theta}_{s}(t) \circ P\right)(X) \quad \text { for } t \in \operatorname{ker} c_{1}, \\
P^{m}(X) & =\bar{\Theta}_{s}\left(m t_{0}\right)(X), \\
\Theta_{s}\left(l t_{0}+t^{\prime}\right)(X) & =\left(P^{l} \circ \bar{\Theta}_{s}\left(t^{\prime}\right)\right)(X) \quad 0 \leq l \leq m-1, t^{\prime} \in \operatorname{ker} c_{1} .
\end{aligned}
$$

Conversely, for every one-parameter group of FPS $\bar{\Theta}_{s}: \operatorname{ker} c_{1} \rightarrow \Gamma^{s}$, $\bar{\Theta}_{s}(t)(X)=X+\sum_{k=2}^{s} \bar{c}_{k}(t) X^{k}$, and for arbitrary $P(X)=\sum_{k=1}^{s} d_{k} X^{k}$ with $d_{1}$ being a primitive root of 1 of order $m$, such that conditions (39)(40) are satisfied, formula (41) properly defines a function $\Theta_{s}: G \rightarrow \Gamma^{s}$, $\Theta_{s}(t)(X)=\sum_{k=1}^{s} c_{k}(t) X^{k}$ and $\Theta_{s}$ is a one-parameter group of FPS with $\operatorname{im} c_{1}=E_{m}$.

Proof. If $\Theta_{s}: G \rightarrow \Gamma^{s}, \Theta_{s}(t)(X)=\sum_{k=1}^{s} c_{k}(t) X^{k}$ is a one-parameter group of FPS, then, clearly, $\left.\Theta_{s}\right|_{\operatorname{ker} c_{1}}=\bar{\Theta}_{s}: \operatorname{ker} c_{1} \rightarrow \Gamma^{s}, \bar{\Theta}_{s}(t)(X)=X+\sum_{k=2}^{s} \bar{c}_{k}(t) X^{k}$ is a one-parameter group of FPS. Put $P(X)=\Theta_{s}\left(t_{0}\right)(X)$. Then $m t_{0} \in \operatorname{ker} c_{1}$,

$$
P^{m}(X)=\Theta_{s}\left(t_{0}\right)^{m}(X)=\Theta_{s}\left(m t_{0}\right)(X)=\bar{\Theta}_{s}\left(m t_{0}\right)(X),
$$

and, since $G$ is a commutative group,

$$
\begin{aligned}
\left(P \circ \bar{\Theta}_{s}(t)\right)(X) & =\left(\Theta_{s}\left(t_{0}\right) \circ \Theta_{s}(t)\right)=\Theta_{s}\left(t_{0}+t\right)(X) \\
& =\Theta_{s}\left(t+t_{0}\right)(X)=\left(\Theta_{s}(t) \circ \Theta_{s}\left(t_{0}\right)\right)(X)=\left(\bar{\Theta}_{s}(t) \circ P\right)(X)
\end{aligned}
$$

for every $t \in \operatorname{ker} c_{1}$. Finally,

$$
\begin{aligned}
\Theta_{s}\left(l t_{0}+t^{\prime}\right)(X) & =\left(\Theta_{s}\left(l t_{0}\right) \circ \Theta_{s}\left(t^{\prime}\right)\right)(X) \\
& =\left(\Theta_{s}\left(t_{0}\right)^{l} \circ \bar{\Theta}_{s}\left(t^{\prime}\right)\right)(X)=\left(P^{l} \circ \bar{\Theta}_{s}\left(t^{\prime}\right)\right)(X)
\end{aligned}
$$

for every $0 \leq l \leq m-1$, and $t^{\prime} \in \operatorname{ker} c_{1}$. 
Now, let us fix a one-parameter group of FPS $\bar{\Theta}_{s}: \operatorname{ker} c_{1} \rightarrow \Gamma_{1}^{s}, \bar{\Theta}_{s}(t)(X)=$ $X+\sum_{k=2}^{s} \bar{c}_{k}(t) X^{k}$, and a FPS $P(X)=\sum_{k=1}^{s} d_{k} X^{k}$, where $d_{1}$ is a primitive root of 1 of the order $m$, satisfying conditions (39)-(40). Then formula (41) properly defines a function $\Theta_{s}: G \rightarrow \Gamma^{s}$. We show that $\Theta_{s}$ is then a oneparameter group of FPS. From (39) it follows that

$$
\left(P^{n} \circ \bar{\Theta}_{s}(t)\right)(X)=\left(\bar{\Theta}_{s}(t) \circ P^{n}\right)(X) \text { for } t \in \operatorname{ker} c_{1},
$$

for every positive integer $n$. Then for $l_{1} t_{0}+t_{1}, l_{2} t_{0}+t_{2} \in G$, where $0 \leq l_{1}, l_{2} \leq$ $m-1$ and $t_{1}, t_{2} \in \operatorname{ker} c_{1}$, using (42) we get

$$
\begin{aligned}
& \left(\left(P^{l_{1}} \circ \bar{\Theta}_{s}\left(t_{1}\right)\right) \circ\left(P^{l_{2}} \circ \bar{\Theta}_{s}\left(t_{2}\right)\right)\right)(X)=\left(P^{l_{1}+l_{2}} \circ \bar{\Theta}_{s}\left(t_{1}+t_{2}\right)\right)(X) \\
& \quad=\left(P^{l_{1}+l_{2}-\left[\frac{l_{1}+l_{2}}{m}\right] m+\left[\frac{l_{1}+l_{2}}{m}\right] m} \bar{\Theta}_{s}\left(t_{1}+t_{2}\right)\right)(X) \\
& =\left(P^{l_{1}+l_{2}-\left[\frac{l_{1}+l_{2}}{m}\right] m} \circ \bar{\Theta}_{s}\left(\left[\frac{l_{1}+l_{2}}{m}\right] m t_{0}+t_{1}+t_{2}\right)\right)(X) \\
& =\Theta_{s}\left(\left(l_{1}+l_{2}-\left[\frac{l_{1}+l_{2}}{m}\right] m\right) t_{0}+\left[\frac{l_{1}+l_{2}}{m}\right] m t_{0}+t_{1}+t_{2}\right)(X) \\
& =\Theta_{s}\left(\left(l_{1} t_{0}+t_{1}\right)+\left(l_{2} t_{0}+t_{2}\right)\right)(X),
\end{aligned}
$$

which finishes the proof.

Proposition 1 proved above gives us only a characterization of the solution of the considered problem in the last case. But in some subcases we are able to give also explicit formulas for one-parameter groups of FPS (i.e. explicit solutions of the system of equations (14)). Note that always $\operatorname{ker} \Theta_{s} \subset \operatorname{ker} c_{1}$. We give the mentioned formulas in the case when $\operatorname{ker} \Theta_{s}=\operatorname{ker} c_{1}$.

Assume once more that im $c_{1}=E_{m}$ with some $m \geq 2$. Then, on account of the first isomorphism theorem (cf. [10, p. 16]), we have $G / \operatorname{ker} c_{1} \cong E_{m}$, and further, since we assumed $\operatorname{ker} \Theta_{s}=\operatorname{ker} c_{1}$,

$$
E_{m} \cong G / \operatorname{ker} c_{1}=G / \operatorname{ker} \Theta_{s} \cong \operatorname{im} \Theta_{s} .
$$

This means that $\operatorname{im} \Theta_{s}$ is a finite subgroup of $\left(\Gamma^{s}, \circ\right)$. From Proposition 1 we deduce then

Corollary 5. Under the assumptions of Proposition 1, if $\Theta_{s}: G \rightarrow$ $\Gamma^{s}, \Theta_{s}(t)(X)=\sum_{k=1}^{s} c_{k}(t) X^{k}$ is a one-parameter group of FPS with $\operatorname{ker} \Theta_{s}=$ $\operatorname{ker} c_{1}$, then there exists a FPS $P(X)=\Theta_{s}\left(t_{0}\right)(X)=\sum_{k=1}^{s} d_{k} X^{k}$ (with $d_{1}=c_{1}\left(t_{0}\right)$ being a primitive root of 1 of order $m$ ) such that

$$
\begin{aligned}
P^{m}(X) & =X, \\
\Theta_{s}\left(l t_{0}+t^{\prime}\right)(X) & =P^{l}(X) \quad 0 \leq l \leq m-1, t^{\prime} \in \operatorname{ker} c_{1} .
\end{aligned}
$$

Conversely, for every FPS $P(X)=\sum_{k=1}^{s} b_{k} X^{k}$ with $d_{1}$ being a primitive root of 1 of order $m$, such that the condition (43) is fulfilled, formula (44) properly defines a function $\Theta_{s}: G \rightarrow \Gamma^{s}, \Theta_{s}(t)(X)=\sum_{k=1}^{s} c_{k}(t) X^{k}$ and $\Theta_{s}$ is a one-parameter group of FPS with im $c_{1}=E_{m}$. 
Proof. It is enough to notice only that the finiteness of $\operatorname{im} \Theta_{s}$ in Proposition 1 implies $\bar{\Theta}_{s}(t)(X)=X$ for $t \in \operatorname{ker} c_{1}$.

In this case every one-parameter group of FPS $\Theta_{s}: G \rightarrow \Gamma^{s}$ must be of the form $\Theta_{s}=\widetilde{\Theta}_{s} \circ \kappa$, where $\kappa: G \rightarrow E_{m}$ is the canonical homomorphism and $\widetilde{\Theta}_{s}: E_{m} \rightarrow \Gamma^{s}, \widetilde{\Theta}_{s}(z)(X)=\sum_{k=1}^{s} \widetilde{c}_{k}(z) X^{k}$ is a one-parameter group of FPS which means that functions $\widetilde{c}_{1}: E_{m} \rightarrow E_{m}$ with im $\widetilde{c}_{1}=E_{m}$, and $\widetilde{c}_{k}: E_{m} \rightarrow \mathbb{K}$ satisfy the system of equations

$$
\left\{\begin{array}{l}
\widetilde{c}_{1}\left(z_{1} z_{2}\right)=\widetilde{c}_{1}\left(z_{1}\right) \widetilde{c}_{1}\left(z_{2}\right) \\
\widetilde{c}_{2}\left(z_{1} z_{2}\right)=\widetilde{c}_{1}\left(z_{1}\right) \widetilde{c}_{2}\left(z_{2}\right)+\widetilde{c}_{2}\left(z_{1}\right) \widetilde{c}_{1}\left(z_{2}\right)^{2} \\
\widetilde{c}_{n}\left(z_{1} z_{2}\right)=\widetilde{c}_{1}\left(z_{1}\right) \widetilde{c}_{n}\left(z_{2}\right)+\widetilde{c}_{n}\left(z_{1}\right) \widetilde{c}_{1}\left(z_{2}\right)^{n} \\
\quad+\sum_{k=2}^{n-1} \widetilde{c}_{k}\left(z_{1}\right) \sum_{\bar{u}_{n} \in U_{n, k}} B_{\bar{u}_{n}} \prod_{j=1}^{n-k+1} \widetilde{c}_{j}\left(z_{2}\right)^{u_{j}}, n \in|3, s|
\end{array}\right.
$$

for $z_{1}, z_{2} \in E_{m}$. We prove

Proposition 2. Let $\widetilde{c}_{1}: E_{m} \rightarrow E_{m}$ be a multiplicative function such that im $\widetilde{c}_{1}=$ $E_{m}$. There exists a sequence of polynomials $\left(P_{n}\right)_{n \geq 2}$ defined by (19) such that for every solution $\left(\widetilde{c}_{n}\right)_{n \in|1, s|}$ of the system of functional equations (45) there exist a sequence of constants $\left(\lambda_{n}\right)_{n \in|2, s|}$ such that for every $n \in|2, s|$

$$
\widetilde{c}_{n}(z)=\lambda_{n}\left(\widetilde{c}_{1}(z)^{n}-\widetilde{c}_{1}(z)\right)+\widetilde{c}_{1}(z) P_{n}\left(\widetilde{c}_{1}(z) ;\left(\lambda_{l}\right)_{l \in|2, n-1|}\right), \quad z \in E_{m} .
$$

Conversely, for each multiplicative function $\widetilde{c}_{1}: E_{m} \rightarrow E_{m}$ and for each sequence $\left(\lambda_{n}\right)_{n \in|2, s|}$ the sequence $\left(\widetilde{c}_{n}\right)_{n \in|2, s|}$ defined by (46) is a solution of the system (45).

Proof. By Theorem 5 we know that $\widetilde{c}_{n}$ defined by (46) for $n \in|2, s|$ satisfy the system of equations (45).

Now, let us consider the second equation of the system (21). Since $m \geq 2$, from Lemma 6 we have

$$
\widetilde{c}_{2}=\lambda_{2}\left(\widetilde{c}_{1}^{2}-\widetilde{c}_{1}\right)=\lambda_{2}\left(\widetilde{c}_{1}^{2}-\widetilde{c}_{1}\right)+\widetilde{c}_{1} P_{2}\left(\widetilde{c}_{1}\right)
$$

with $P_{2}(X)=0$ and some $\lambda_{2} \in \mathbb{K}$. Then, analogously as in the proof of Theorem 5, assume that for some $n \in|3, s|$ we have

$$
\widetilde{c}_{j}(z)=\lambda_{n}\left(\widetilde{c}_{1}(z)^{j}-\widetilde{c}_{1}(z)\right)+\widetilde{c}_{1}(z) P_{j}\left(\widetilde{c}_{1}(z) ;\left(\lambda_{l}\right)_{l \in|2, j-1|}\right), \quad z \in E_{m},
$$

for every $j \in|2, n-1|$, and consider the equation of the system (45) with the function $\widetilde{c}_{n}$ on the left hand side, i.e.

$$
\begin{aligned}
\widetilde{c}_{n}\left(z_{1} z_{2}\right)= & \widetilde{c}_{1}\left(z_{1}\right) \widetilde{c}_{n}\left(z_{2}\right)+\widetilde{c}_{n}\left(z_{1}\right) \widetilde{c}_{1}\left(z_{2}\right)^{n} \\
& +\sum_{k=2}^{n-1} \widetilde{c}_{k}\left(z_{1}\right) \sum_{\bar{u}_{n} \in U_{n, k}} B_{\bar{u}_{n}} \prod_{j=1}^{n-k+1} \widetilde{c}_{j}\left(z_{2}\right)^{u_{j}}, \quad z_{1}, z_{2} \in E_{m} .
\end{aligned}
$$


The procedure of finding the solution of (47) is almost the same as in Theorem 5 (cf. the solution of Eq. (38)). Note that every solution of Eq. (47) is the sum of a particular solution of this equation (i.e. $\widetilde{c}_{1}(z) P_{n}\left(\widetilde{c}_{1}(z) ;\left(\lambda_{l}\right)_{l \in|2, n-1|}\right)$ ), and a solution of the equation

$$
f\left(z_{1} z_{2}\right)=c_{1}\left(z_{1}\right) f\left(z_{2}\right)+c_{1}\left(z_{2}\right)^{n} f\left(z_{1}\right) \text { for } z_{1}, z_{2} \in E_{m} .
$$

Let us consider two cases

1. $n \not \equiv 1 \bmod m$,

2. $n \equiv 1 \bmod m$.

In the first case, as in the proof of Theorem 5 (then one can easily find $z_{0} \in E_{m}$ such that $\left.\widetilde{c}_{1}\left(z_{0}\right)^{n}-\widetilde{c}_{1}\left(z_{0}\right) \neq 0\right)$ we show that

$$
\widetilde{c}_{n}(z)=\lambda_{n}\left(\widetilde{c}_{1}(z)^{n}-\widetilde{c}_{1}(z)\right)+\widetilde{c}_{1}(z) P_{n}\left(\widetilde{c}_{1}(z) ;\left(\lambda_{l}\right)_{l \in|2, n-1|}\right), \quad z \in E_{m} .
$$

Now we will show that also in the second case the same formula holds, even if it has in some sense a different meaning. Note that for $n \equiv 1 \bmod m$, since $\operatorname{im} \widetilde{c}_{1}=E_{m}$, we have $\widetilde{c}_{1}(z)^{n}=\widetilde{c}_{1}(z)$ for $z \in E_{m}$. Thus, from (48) we obtain

$$
f\left(z_{1} z_{2}\right)=\widetilde{c}_{1}\left(z_{1}\right) f\left(z_{2}\right)+\widetilde{c}_{1}\left(z_{2}\right) f\left(z_{1}\right) \text { for } z_{1}, z_{2} \in E_{m} .
$$

It is easy to see that if a function $f: E_{m} \rightarrow \mathbb{K}$ satisfies (49), then $l: E_{m} \rightarrow \mathbb{K}$, $l(z)=\frac{f(z)}{\widetilde{c}_{1}(z)}$ for $z \in E_{m}$, is a generalized logarithmic function, and since $E_{m}$ is finite, $l=0$. Hence $f=0$. Thus each solution of Eq. (47) in the second case must be of the form

$$
\begin{aligned}
\widetilde{c}_{n}(z) & =\widetilde{c}_{1}(z) P_{n}\left(\widetilde{c}_{1}(z) ;\left(\lambda_{l}\right)_{l \in|2, n-1|}\right) \\
& =\lambda_{n}\left(\widetilde{c}_{1}(z)^{n}-\widetilde{c}_{1}(z)\right)+\widetilde{c}_{1}(t) P_{n}\left(\widetilde{c}_{1}(t) ;\left(\lambda_{l}\right)_{l \in|2, n-1|}\right),
\end{aligned}
$$

which finishes the proof.

Corollary 6. Let $(G,+)$ be a commutative group and let a sequence of polynomials $\left(P_{n}\right)_{n \in|2, s|}$ be given by (19). Every one-parameter group $\Theta(t)(X)=$ $\sum_{k=1}^{s} c_{k}(t) X^{k}$ of FPS such that $\operatorname{im} c_{1}=E_{m}$ and $\operatorname{ker} \Theta_{s}=\operatorname{ker} c_{1}$ is given by (37), where $\left(\lambda_{n}\right)_{n \in|2, s|}$ is an arbitrary sequence of constants.

Proof. We know that if im $c_{1}=E_{m}$ and $\operatorname{ker} \Theta_{s}=\operatorname{ker} c_{1}$ then $\Theta_{s}=\widetilde{\Theta}_{s} \circ \kappa$, where $\kappa: G \rightarrow E_{m}$ is the canonical homomorphism and $\widetilde{\Theta}_{s}: E_{m} \rightarrow \Gamma^{s}$, $\widetilde{\Theta}_{s}(z)(X)=\sum_{k=1}^{s} \widetilde{c}_{k}(z) X^{k}$ is a one-parameter group of FPS. Thus $c_{n}=\widetilde{c}_{n} \circ \kappa$ for every $n \in|1, s|$. On account of Proposition 2, $c_{n}=\widetilde{c}_{n} \circ \kappa$ for $n \in|1, s|$, so for every $t \in G$ we have

$$
\begin{aligned}
c_{n}(t) & =\left(\widetilde{c}_{n} \circ \kappa\right)(t) \\
& =\lambda_{n}\left(\left(\widetilde{c}_{1} \circ \kappa\right)(t)^{n}-\left(\widetilde{c}_{1} \circ \kappa\right)(t)\right)+\left(\widetilde{c}_{1} \circ \kappa\right)(t) P_{n}\left(\left(\widetilde{c}_{1} \circ \kappa\right)(t) ;\left(\lambda_{l}\right)_{l \in|2, n-1|}\right) \\
& =\lambda_{n}\left(c_{1}(t)^{n}-c_{1}(t)\right)+c_{1}(t) P_{n}\left(c_{1}(t) ;\left(\lambda_{l}\right)_{l \in|2, n-1|}\right) .
\end{aligned}
$$

This finishes the proof. 


\section{Iterative roots of unit in $\Gamma^{s}$}

Using results of the previous section we describe iterative roots of $X$ in rings of FPS and truncated FPS. Let $s$ be a positive integer or $s=\infty$. It is easy to see that for a fixed positive integer $m \geq 2$, a power series $P(X)=\sum_{j=1}^{s} d_{j} X^{j}$ is an iterative root of $X$ of order $m$ if and only if $\Theta_{E_{m}}: E_{m} \rightarrow \Gamma^{s}$,

$$
\Theta_{E_{m}}\left(e^{\frac{2 l \pi i}{m}}\right)=P^{l}(X) \quad \text { for } l \in\{0,1, \ldots, m-1\}
$$

is a one-parameter group of truncated FPS. Thus the iterative roots of $X$ in the ring of FPS lie in an image of one-parameter groups of formal power series $\Theta_{E_{m}}(t)(X)=\sum_{k=1}^{s} c_{k}(t) X^{k}, t \in E_{m}$.

First, note that $c_{1} \neq 1$. Indeed, if $c_{1}=1$, then for $(G,+)=\left(E_{m}, \cdot\right)$, every homomorphism $a: G \rightarrow \mathbb{K}$ on a finite group is the zero function (every logarithmic function $a: E_{m} \rightarrow \mathbb{K}$ is the zero function), so (cf. (46)) $\Theta_{E_{m}}(t)(X)=X$ for every $t \in G$.

Finally, to describe all iterative roots it is enough to take $c_{1}=\mathrm{id}_{E_{m}}$, since in the case $c_{1} \neq 1$ all functions $c_{k}$ with $k \in|2, s|$ are polynomials in $c_{1}$ and for every homomorphism $c_{1}: E_{m} \rightarrow E_{m}$ we have $\operatorname{im} c_{1} \subset \operatorname{imid} E_{E_{m}}$. In [8] we proved a description of one-parameter groups of FPS using simultaneous conjugation, namely

Lemma 7. (cf. [8, Theorem 5]) Let $c_{1}: E_{m} \rightarrow \mathbb{K} \backslash\{0\}, c_{1} \neq 1$ be a generalized exponential function. Every one-parameter group of FPS $F^{[s]}(t, X)_{t \in E_{m}}$, $F^{[s]}(t, X)=\sum_{k=1}^{s} c_{k}(t) X^{k}$ with $c_{k}: E_{m} \rightarrow \mathbb{K}$ for $k \in|2, s|$, is given by

$$
F^{[s]}(t, X)=U^{-1}\left(c_{1}(t) U(x)\right) \text { for every } t \in E_{m},
$$

where $U(X) \in \Gamma_{1}^{s}$ is an arbitrary invertible formal power series with respect to $\circ$. Thus every (primitive) root $P(X)$ of $X$ in $\Gamma^{s}$ is given by

$$
P(X)=U^{-1}\left(e^{\frac{2 l_{0} \pi i}{m}} U(X)\right)
$$

where $0 \leq l_{0} \leq m-1\left(\right.$ and $\operatorname{gcd}\left(l_{0}, m\right)=1$ if $P(X)$ has order $m$ in the group $\left.\Gamma^{s}\right)$.

\section{One-parameter groups with infinite image}

Using Proposition 1 we are able to give now a description of the one-parameter groups of FPS in the case $\operatorname{im} c_{1}=E_{m}$ under an additional assumption on $G$. We use in our description semicanonical forms (for the definition of $\mathcal{N}_{m}^{s}$ see the Sect. 2). We begin with

Lemma 8. Assume that $\widetilde{\Theta}_{s}: G \rightarrow \Gamma_{1}^{s}, \widetilde{\Theta}_{s}(t)(X)=X+\sum_{k=p+2}^{s} \widetilde{c}_{k}(t) X^{k}$, $\widetilde{c}_{p+2} \neq 0$, is a one-parameter group of FPS (which means that $\left(\widetilde{c}_{k}\right)_{k \in|p+2, s|}$ are given by (32)) and fix an integer $m \geq 2$. Then $\operatorname{im} \widetilde{\Theta}_{s} \subset \mathcal{N}_{m}^{s}$ if and only if 
$p \geq 1, p+2=q m+1$ with an integer $q \geq 1, h_{n}=0$ for $n \in|q m+2, s-q m| \backslash \mathbb{N}_{m}$ and $a_{n}=0$ for $n \in|s-(q m-1), s| \backslash \mathbb{N}_{m}$. In this case we have with additive functions $a, a_{k m+1}: G \rightarrow \mathbb{K}$

$$
\begin{aligned}
\widetilde{c}_{q m+1}(t)= & a(t), \quad t \in G \\
\widetilde{c}_{k m+1}(t)= & h_{k m+1} a(t) \\
& +\widetilde{L}_{k m+1}^{m, q}\left(a(t),\left(h_{j m+1}\right)_{j \in|q+1, k-q|}\right), \quad t \in G, k \in|q+1, r-q|, \\
\widetilde{c}_{k m+1}(t)= & a_{k m+1}(t) \\
& +\widetilde{L}_{k m+1}^{m, q}\left(a(t),\left(h_{j p+1}\right)_{j \in|q+1, k-q|}\right), \quad t \in G, k \in|r-q, r|,
\end{aligned}
$$

with $h_{q m+1}=1$ and

$$
\left\{\begin{array}{l}
\widetilde{L}_{q m+1}^{m, q}(X)=0, \\
\widetilde{L}_{k m+1}^{m, q}\left(X ;\left(h_{j m+1}\right)_{j \in|q+1, k-q|}\right)=\sum_{l=q}^{k-q} h_{l m+1} \sum_{\bar{u}_{k m+1} \in U_{k m+1, l m+1}^{m, q}} B_{\bar{u}_{k m+1}} \\
\quad \times \int_{0}^{X}\left(\prod_{j=q}^{k-l}\left(h_{j m+1} s+\widetilde{L}_{j m+1}^{m, q}\left(s ;\left(h_{n m+1}\right)_{n \in|q+1, j-q|}\right)\right)^{u_{j p+1}}\right) d s .
\end{array}\right.
$$

Proof. Let $\widetilde{\Theta}_{s}: G \rightarrow \Gamma_{1}^{s}, \widetilde{\Theta}_{s}(t)(X)=X+\sum_{k=p+2}^{s} \widetilde{c}_{k}(t) X^{k}, \widetilde{c}_{p+2} \neq 0$, be a one-parameter group of FPS. From Theorem 4 we derive that there exist a nonzero additive function $a: G \rightarrow \mathbb{K}$, a sequence of additive functions $\left(a_{n}\right)_{n \in|s-p, s|}, a_{n}: G \rightarrow \mathbb{K}$, and a sequence of constants $\left(h_{n}\right)_{n \in|p+3, s-p-1|}$ such that the coefficient functions $\left(\widetilde{c}_{k}\right)_{k \in|p+2, s|}$ are given by (32), i.e.

$$
\begin{aligned}
& \widetilde{c}_{p+2}(t)=a(t), t \in G \\
& \widetilde{c}_{n}(t)=h_{n} a(t)+\bar{L}_{n}^{p}\left(a(t) ;\left(h_{j}\right)_{j \in|p+3, n-p-1|}\right), t \in G, n \in|p+3, s-p-1|, \\
& \left.\widetilde{c}_{n}(t)=a_{n}(t)+\bar{L}_{n}^{p}\left(a(t) ;\left(h_{j}\right)_{j \in|p+3, n-p-1|}\right)\right), t \in G, n \in|s-p, s|,
\end{aligned}
$$

where the polynomials $\left(\bar{L}_{n}^{p}\right)_{n \in|p+3, s|}$ are defined by (28) and (18). From the definition of $\mathcal{N}_{m}^{s}$ we derive that im $\Theta_{s} \subset \mathcal{N}_{m}^{s}$ only if $p \geq 1$ and $p+2=q m+1$ with some positive integer $q$.

First we show that if $h_{n}=0$ for $n \in|p+3, s-p-1| \backslash \mathbb{N}_{m}$ and $a_{n}=0$ for $n \in|s-p, s| \backslash \mathbb{N}_{m}$, then $\widetilde{c}_{n}=0$ for $n \in|p+3, s| \backslash \mathbb{N}_{m}$. Since $k=p+2>$ $2=(p+3)-p-1$, by $(18)$ we obtain $\bar{L}_{p+3}^{p}(X)=0$. Moreover, $p+3 \notin \mathbb{N}_{m}$ implies $h_{p+3}=0$. Hence $\widetilde{c}_{p+3}=h_{p+3} a+L_{p+3}^{p}(a)=0$. Assume that for some $n \in|p+4, s-p-1| \backslash \mathbb{N}_{m}$,

$$
\widetilde{c}_{k}=h_{k} a+\bar{L}_{k}^{p}\left(a ;\left(h_{j}\right)_{j \in|p+3, k-p-1|}\right)=0 \quad \text { for } k \in|p+3, n-1| \backslash \mathbb{N}_{m} .
$$

We prove that $\widetilde{c}_{n}=h_{n} a+\bar{L}_{n}^{p}\left(a ;\left(h_{l}\right)_{l \in|p+3, n-p-1|}\right)=0$. Indeed, with $h_{p+2}=1$, on account of (52) and the definition of $\bar{L}_{n}^{p}$ we have 


$$
\begin{aligned}
\widetilde{c}_{n}(t)= & h_{n} a(t)+\bar{L}_{n}^{p}\left(a(t) ;\left(h_{j}\right)_{j \in|p+3, n-p-1|}\right) \\
= & h_{n} a(t)+\sum_{k=p+2}^{n-p-1} h_{k} \sum_{\bar{u}_{n} \in U_{n, k}^{p}} B_{\bar{u}_{n}} \\
& \times \int_{0}^{a(t)}\left(\prod_{j=p+2}^{n-k+1}\left(h_{j} s+\bar{L}_{j}^{p}\left(s ;\left(h_{l}\right)_{l \in|p+3, j-p-1|}\right)\right)^{u_{j}}\right) d s .
\end{aligned}
$$

Since $h_{k}=0$ for $k \in|p+2, n-p-1| \backslash \mathbb{N}_{m}$ and $\widetilde{c}_{k}=h_{k} a+$ $\bar{L}_{k}^{p}\left(a ;\left(h_{j}\right)_{j \in|p+3, k-p-1|}\right)=0$ for $k \in|p+3, n-1| \backslash \mathbb{N}_{m}$, by Corollaries 2 and 3 (i) we get

$$
\widetilde{c}_{n}=h_{n} a+\bar{L}_{n}^{p}\left(a ;\left(h_{l}\right)_{l \in|p+3, n-p-1|}\right)=0 .
$$

Finally, assume that

$$
\begin{aligned}
& \widetilde{c}_{k}=h_{k} a+\bar{L}_{k}^{p}\left(a ;\left(h_{j}\right)_{j \in|p+3, k-p-1|}\right)=0 \text { for } k \in|p+3, s-p-1| \backslash \mathbb{N}_{m}, \\
& \widetilde{c}_{k}=a_{k}+\bar{L}_{k}^{p}\left(a ;\left(h_{j}\right)_{j \in|p+3, k-p-1|}\right)=0 \text { for } k \in|s-p, n-1| \backslash \mathbb{N}_{m},
\end{aligned}
$$

with some $n \in|s-p, s| \backslash \mathbb{N}_{m}$. In the same way as above we can show that $\widetilde{c}_{n}=a_{n}+\bar{L}_{n}^{p}\left(a ;\left(h_{j}\right)_{j \in|p+3, n-p-1|}\right)=0$.

Conversely, let $p+2=q m+1$ with $q \in \mathbb{N}$ and

$$
\begin{aligned}
& \widetilde{c}_{k}=h_{k} a+\bar{L}_{k}^{p}\left(a ;\left(h_{j}\right)_{j \in|p+3, k-p-1|}\right)=0 \quad \text { for } k \in|p+3, s-p-1| \backslash \mathbb{N}_{m}, \\
& \widetilde{c}_{k}=a_{k}+\bar{L}_{k}^{p}\left(a ;\left(h_{j}\right)_{j \in|p+3, k-p-1|}\right)=0 \quad \text { for } k \in|s-p, s| \backslash \mathbb{N}_{m} .
\end{aligned}
$$

We show that $h_{n}=0$ for $n \in|p+3, s-p-1| \backslash \mathbb{N}_{m}$ and $a_{n}=0$ for $n \in|s-p, s| \backslash$ $\mathbb{N}_{m}$. Indeed, since $p+3 \notin \mathbb{N}_{m}$ and $\bar{L}_{p+3}^{p}(X)=0,0=c_{p+3}=h_{p+3} a+\bar{L}_{p+3}^{p}(a)$ implies $h_{p+3}=0$. Assume next that for some $n \in|p+4, s-p-1| \backslash \mathbb{N}_{m}$,

$$
h_{k}=0 \quad \text { for every } k \in|p+3, n-1| \backslash \mathbb{N}_{m} .
$$

We show that $h_{n}=0$. From the definition of $\bar{L}_{n}^{p}$ and our assumptions (53) and (54), on account of Corollaries 2 and 3 (i) we have $\left(h_{p+2}=1\right)$

$$
\begin{aligned}
& \bar{L}_{n}^{p}\left(a(t) ;\left(h_{l}\right)_{l \in|p+3, n-p-1|}\right) \\
& =\sum_{k=p+2}^{n-p-1} h_{k} \sum_{\bar{u}_{n} \in U_{n, k}^{p}} B_{\bar{u}_{n}} \int_{0}^{a(t)}\left(\prod_{j=p+2}^{n-k+1}\left(h_{j} s+\bar{L}_{j}^{p}\left(s ;\left(h_{r}\right)_{r \in|p+3, j-p-1|}\right)\right)^{u_{j}}\right) d s \\
& =\sum_{k=p+2}^{n-p-1} h_{k} \sum_{\bar{u}_{n} \in U_{n, k}^{q, m}} B_{\bar{u}_{n}} \int_{0}^{a(t)}\left(\prod_{j=p+2}^{n-k+1}\left(h_{j} s+\bar{L}_{j}^{p}\left(s ;\left(h_{r}\right)_{r \in|p+3, j-p-1|}\right)\right)^{u_{j}}\right) d s \\
& =0 .
\end{aligned}
$$


Consequently, by (53)

$$
0=\widetilde{c}_{n}=h_{n} a(t)+\bar{L}_{n}^{p}\left(a(t) ;\left(h_{l}\right)_{l \in|p+3, n-p-1|}\right)=h_{n} a(t),
$$

which with $a \neq 0$ implies $h_{n}=0$.

Next, let

$$
\begin{array}{ll}
h_{k}=0 & \text { for } k \in|p+3, s-p-1| \backslash \mathbb{N}_{m}, \\
a_{k}=0 & \text { for } k \in|s-p, n-1| \backslash \mathbb{N}_{m},
\end{array}
$$

with some $n \in|s-p, s| \backslash \mathbb{N}_{m}$. In the same way as above we show that

$$
0=c_{n}=a_{n}+\bar{L}_{n}^{p}\left(a ;\left(h_{j}\right)_{j \in|p+3, n-p-1|}\right)=a_{n} .
$$

Finally, let $p+2=q m+1$ with $q \in \mathbb{N}$ and assume that $\widetilde{\Theta}_{s}: G \rightarrow \Gamma_{1}^{s}$, $\widetilde{\Theta}_{s}(t)(X)=X+\sum_{k=p+2}^{s} \widetilde{c}_{k}(t) X^{k}, \widetilde{c}_{p+2} \neq 0$, be a one-parameter group of FPS satisfying $\operatorname{im} \widetilde{\Theta}^{s} \subset \mathcal{N}_{m}^{s}$ where the sequence of functions $\left(\widetilde{c}_{k}\right)_{k \in|p+2, s|}$ is given by (52) with

$$
\begin{aligned}
& h_{n}=0 \text { for } n \in|q m+2, s-q m| \backslash \mathbb{N}_{m}, \\
& a_{n}=0 \text { for } n \in|s-(q m-1), s| \backslash \mathbb{N}_{m} .
\end{aligned}
$$

Then we obtain (50) with polynomials $\widetilde{L}_{k m+1}^{m, q}$ defined as follows. By our assumption we have $p=q m-1$. Put

$$
\widetilde{L}_{q m+1}^{m, q}(X):=\bar{L}_{p+2}^{p}(X)=X .
$$

Further, from the definition of the sequence of polynomials $\left(\bar{L}_{n}^{p}\right)_{n \in|p+2, s|}$ and (55), using Corollary 3 (ii) we define

$$
\begin{aligned}
\widetilde{L}_{k m+1}^{m, q}\left(X ;\left(h_{j m+1}\right)_{j \in|q+1, k-q|}\right)=L_{k m+1}^{q m-1}\left(X ;\left(h_{j}\right)_{j \in|q m+1,(k-q) m+1|}\right) \\
=\sum_{l=q m+1}^{(k-q) m+1} h_{l} \sum_{\bar{u}_{k m+1} \in U_{k m+1, l}^{q m-1}} B_{\bar{u}_{k m+1}} \\
\quad \times \int_{0}^{X}\left(\prod_{j=q m+1}^{k m+1-l+1}\left(h_{j} s+L_{j}^{p}\left(s ;\left(h_{r}\right)_{r \in|q m+1, j-q m|}\right)\right)^{u_{j}}\right) d s \\
=\sum_{l=q}^{k-q} h_{l m+1} B_{\bar{u}_{k m+1}} \sum_{\bar{u}_{k m+1} \in U_{k m+1, l m+1}^{m, q}}{ }^{X}\left(\prod_{0}^{k-l}\left(h_{j m+1} s+L_{j m+1}^{m, q}\left(s ;\left(h_{n m+1}\right)_{n \in|q+1, j-q|}\right)\right)^{u_{j p+1}}\right) d s
\end{aligned}
$$

which finishes the proof. 
We are able now to give a description of the one-parameter groups of FPS in the case $\operatorname{im} c_{1}=E_{m}$ under the additional assumption on the group $G$. We prove

Theorem 6. Let $s$ be either a positive integer or $s=\infty$ and let $\Theta_{s}: G \rightarrow$ $\Gamma^{s}, \Theta_{s}(t)(X)=\sum_{k=1}^{s} c_{k}(t) X^{k}$ be a one-parameter group of FPS. Assume that $(G,+)$ is a commutative group and $c_{1}: G \rightarrow \mathbb{K} \backslash\{0\}$ is a generalized exponential function such that im $c_{1}=E_{m}$ with an integer $m \geq 2$. Moreover, let $t_{0} \in G$ be such that $c_{1}\left(t_{0}\right)$ is a primitive root of 1 of order $m$ and $\left\{l t_{0}: 0 \leq l \leq m-1\right\}$ is a subgroup of $(G,+)$ (then $m t_{0}=0 \in \operatorname{ker} c_{1}$, every $t \in G$ may be uniquely written as $l t_{0}+t^{\prime}$ with some $0 \leq l \leq m-1, t^{\prime} \in \operatorname{ker} c_{1}$ and $G / \operatorname{ker} c_{1}=$ $\left.\left\{l t_{0}+\operatorname{ker} c_{1}: 0 \leq l \leq m-1\right\} \cong\left\{l t_{0}: 0 \leq l \leq m-1\right\}\right)$.

Then there exists a FPS $U(X)=X+\sum_{k=2}^{s} v_{k} X^{k} \in \Gamma_{1}^{s}$ such that

$$
\begin{aligned}
\left(U \circ \Theta_{s}(t) \circ U^{-1}\right)(X) & =\left(U \circ \Theta_{s}\left(l t_{0}+t^{\prime}\right) \circ U^{-1}\right)(X) \\
& =e^{\frac{2\left(l_{0}+l\right) \pi i}{m}} \widetilde{\Theta}_{s}\left(t^{\prime}\right) \\
& =e^{\frac{2\left(l_{0}+l\right) \pi i}{m}}\left(X+\sum_{k=q}^{r} \widetilde{c}_{k m+1}\left(t^{\prime}\right) X^{k m+1}\right),
\end{aligned}
$$

with a fixed $0 \leq l_{0} \leq m-1, \operatorname{gcd}\left(l_{0}, m\right)=1$, where $q \geq 1$ is an integer, $r=\infty$ if $s=\infty$ and $r m+1 \leq s<(r+1) m+1$ otherwise, the functions $\widetilde{c}_{k m+1}$ for $k \in|q, r|$ are given by $(50)$, where $\left(\widetilde{L}_{k m+1}^{m, q}\right)_{k \in|q, r|}$ is given by (51), $\left(h_{j m+1}\right)_{j \in|q+1, r-q|}$ is a sequence of constants, $\left(a_{j m+1}\right)_{j \in|r-q+1, r|}, a_{j m+1}: G \rightarrow$ $\mathbb{K}$ are additive functions. Then we may write (56) equivalently as

$$
\left\{\begin{array}{l}
c_{1}\left(l t_{0}+t^{\prime}\right)=e^{\frac{2\left(l_{0}+l\right) \pi i}{m}}, \\
c_{n}\left(k t_{0}+t^{\prime}\right)= \\
e^{\frac{2\left(l_{0}+l\right) \pi i}{m}}\left(v_{n}+\sum_{k \in|q m+1, n-1| \cap \mathbb{N}_{m}} \widetilde{c}_{k}\left(t^{\prime}\right) \sum_{\bar{u}_{n} \in U_{n, k}} B_{\bar{u}_{n}} \prod_{j=2}^{n-k+1} v_{j}^{u_{j}}+\widetilde{c}_{n}\left(t^{\prime}\right)\right) \\
\quad-\sum_{k=2}^{n-1} v_{k} \sum_{\bar{u}_{n} \in U_{n, k}} B_{\bar{u}_{n}} \prod_{j=1}^{n-k+1} c_{j}\left(l t_{0}+t^{\prime}\right)^{u_{j}}-e^{\frac{2\left(l_{0}+l\right) n \pi i}{m}} v_{n}, n \in|2, s|,
\end{array}\right.
$$

with $\widetilde{c}_{k}\left(t^{\prime}\right)=0$ for $k \in|2, q m| \cup\left(|q m+2, s| \backslash \mathbb{N}_{m}\right)$.

Conversely, for every FPS $U(X)=X+\sum_{k=2}^{s} v_{k} X^{k} \in \Gamma_{1}^{s}$, and for an arbitrary one-parameter group of FPS $\widetilde{\Theta}_{s}: \operatorname{ker} c_{1} \rightarrow \Gamma_{1}^{s}$ given by (50), where $q \geq 1$ is an arbitrary integer, $\left(\widetilde{L}_{k m+1}^{m, q}\right)_{k \in|q, r|}$ is given by $(51),\left(h_{j m+1}\right)_{j \in|q+1, r-q|}$ is a sequence of constants, $\left(a_{j m+1}\right)_{j \in|r-q+1, r|}, a_{j m+1}: G \rightarrow \mathbb{K}$ are additive functions, the formulas (56) or (57) properly define a function $\Theta_{s}: G \rightarrow \Gamma^{s}$, $\Theta_{s}(t)(X)=\sum_{k=1}^{s} c_{k}(t) X^{k}$ and $\Theta_{s}$ is a one-parameter group of FPS with $\operatorname{im} c_{1}=E_{m}$. 
Proof. Let $\Theta_{s}: G \rightarrow \Gamma^{s}, \Theta_{s}(t)(X)=\sum_{k=1}^{s} c_{k}(t) X^{k}$ be a one-parameter group of FPS. On account of Proposition 1, $\left.\Theta_{s}\right|_{\operatorname{ker} c_{1}}=\bar{\Theta}_{s}: \operatorname{ker} c_{1} \rightarrow \Gamma_{1}^{s}, \bar{\Theta}_{s}(t)(X)=$ $\sum_{k=1}^{s} \bar{c}_{k}(t) X^{k}$, is a one-parameter group with $\bar{c}_{1}=1$ and $P(X)=\Theta_{s}\left(t_{0}\right)(X)=$ $\sum_{k=1}^{s} d_{k} X^{k}\left(d_{1}=c_{1}\left(t_{0}\right)\right.$ is a primitive root of 1 of order $\left.m\right)$ such that (39)(40) hold. Since $m t_{0}=0$, we have $P^{m}(X)=\bar{\Theta}_{s}(0)(X)=X$ and hence $P(X)$ is a root of $X$ in $\Gamma^{s}$ of order $m$. By Lemma $7, P(X)=\left(U^{-1} \circ L_{\rho} \circ U\right)(X)$, where $\rho=e^{\frac{2 l_{0} \pi i}{m}}, L_{\rho}(X)=\rho X, 0 \leq l_{0} \leq m-1, \operatorname{gcd}\left(l_{0}, m\right)=1$ and $U(X)=$ $X+\sum_{j=2}^{s} v_{j} X^{j} \in \Gamma_{1}^{s}$. Then, from (39) we obtain

$$
\left(\left[U^{-1} \circ L_{\rho} \circ U\right] \circ \bar{\Theta}_{s}\left(t^{\prime}\right)\right)(X)=\left(\bar{\Theta}_{s}(t) \circ\left[U^{-1} \circ L_{\rho} \circ U\right]\right)
$$

for every $t^{\prime} \in \operatorname{ker} c_{1}$, or, equivalently

$$
e^{\frac{2 l_{0} \pi i}{m}}\left(U \circ \bar{\Theta}_{s}\left(t^{\prime}\right) \circ U^{-1}\right)(X)=\left(U \circ \bar{\Theta}_{s}\left(t^{\prime}\right) \circ U^{-1}\right)\left(e^{\frac{2 l_{0} \pi i}{m}} X\right) .
$$

This implies $\left(U \circ \bar{\Theta}_{s}\left(t^{\prime}\right) \circ U^{-1}\right)(X) \in \mathcal{N}_{m}^{s}$ for every $t^{\prime} \in \operatorname{ker} c_{1}$. Hence

$$
\left(U \circ \bar{\Theta}_{s}\left(t^{\prime}\right) \circ U^{-1}\right)(X)=\widetilde{\Theta}_{s}\left(t^{\prime}\right)(X)=X+\sum_{k=q}^{r} \widetilde{c}_{k m+1}\left(t^{\prime}\right) X^{k m+1},
$$

where $q \geq 1$ is an integer and either $r=\infty$ if $s=\infty$ or $r m+1 \leq s<$ $(r+1) m+1$ provided $s<\infty$. From the last equality it follows that $\widetilde{\Theta}_{s}$ : $\operatorname{ker} c_{1} \rightarrow \mathcal{N}_{m}^{s}, \widetilde{\Theta}_{s}\left(t^{\prime}\right)(X)=X+\sum_{k=q}^{r} \widetilde{c}_{k m+1}\left(t^{\prime}\right) X^{k m+1}$ is a one-parameter group of FPS, hence by Lemma 8 , the functions $\widetilde{c}_{k m+1}$ are given by (50), where $\left(\widetilde{L}_{k m+1}^{m, q}\right)_{k \in|q, r|}$ is given by $(51),\left(h_{j m+1}\right)_{j \in|q+1, r-q|}$ is a sequence of constants, $\left(a_{j m+1}\right)_{j \in|r-q+1, r|}, a_{j m+1}: G \rightarrow \mathbb{K}$ are additive functions. Then, from (41) we obtain

$$
\begin{aligned}
\Theta_{s}(t)(X) & =\Theta_{s}\left(l t_{0}+t^{\prime}\right)(X)=\left(P^{l} \circ \bar{\Theta}_{s}\left(t^{\prime}\right)\right)(X) \\
& =\left(\left[U^{-1} \circ L_{\rho} \circ U\right]^{l} \circ\left[U^{-1} \circ \widetilde{\Theta}_{s}\left(t^{\prime}\right) \circ U\right]\right)(X) \\
& =\left(\left[U^{-1} \circ L_{\rho^{l}} \circ U\right] \circ\left[U^{-1} \circ \widetilde{\Theta}_{s}\left(t^{\prime}\right) \circ U\right]\right)(X)
\end{aligned}
$$

which proves (56). In particular,

$$
\left(U \circ \Theta_{s}\left(l t_{0}+t^{\prime}\right)\right)(X)=e^{\frac{2\left(l_{0}+l\right) \pi i}{m}}\left(\widetilde{\Theta}_{s}\left(t^{\prime}\right) \circ U\right)(X),
$$

which gives $c_{1}\left(l t_{0}+t^{\prime}\right)=e^{\frac{2\left(l_{0}+l\right) \pi i}{m}}$ and

$$
\begin{aligned}
& c_{n}\left(l t_{0}+t^{\prime}\right)+\sum_{k=2}^{n-1} v_{k} \sum_{\bar{u}_{n} \in U_{n, k}} B_{\bar{u}_{n}} \prod_{j=1}^{n-k+1} c_{j}\left(l t_{0}+t^{\prime}\right)^{u_{j}}+e^{\frac{2\left(l_{0}+l\right) n \pi i}{m}} v_{n} \\
& =e^{\frac{2\left(l_{0}+l\right) \pi i}{m}}\left(v_{n}+\sum_{k \in|q m+1, n-1| \cap \mathbb{N}_{m}} \widetilde{c}_{k}\left(t^{\prime}\right) \sum_{\bar{u}_{n} \in U_{n, k}} B_{\bar{u}_{n}} \prod_{j=2}^{n-k+1} v_{j}^{u_{j}}+\widetilde{c}_{n}\left(t^{\prime}\right)\right),
\end{aligned}
$$


for $n \in|2, s|$ with $\widetilde{c}_{k}\left(t^{\prime}\right)=0$ for $k \in|2, q m| \cup\left(|q m+2, s| \backslash \mathbb{N}_{m}\right)$. This proves (57).

The converse is a simple conclusion from Proposition 1. Indeed, if $U(X)=$ $X+\sum_{k=2}^{s} v_{k} X^{k} \in \Gamma_{1}^{s}$ is a FPS and $\widetilde{\Theta}_{s}: \operatorname{ker} c_{1} \rightarrow \Gamma_{1}^{s}$ is a one-parameter group of FPS given by (50), then $\bar{\Theta}_{s}: \operatorname{ker} c_{1} \rightarrow \Gamma_{1}^{s}$,

$$
\bar{\Theta}_{s}(t)(X)=\left(U^{-1} \circ \widetilde{\Theta}_{s}(t) \circ U\right)(X) \text { for } t \in \operatorname{ker} c_{1},
$$

is a one-parameter group of FPS, $P(X)=\left(U^{-1} \circ L_{\rho} \circ U\right)(X)$, where $\rho=$ $e^{\frac{2 l_{0} \pi i}{m}}, L_{\rho}(X)=\rho X, 0 \leq l_{0} \leq m-1, \operatorname{gcd}\left(l_{0}, m\right)=1$, commutes with $\bar{\Theta}_{s}\left(t^{\prime}\right)$ and it is a root of $X$ in $\Gamma^{s}$ of order $m$ (note that $\left.\bar{\Theta}_{s}\left(m t_{0}\right)(X)=\bar{\Theta}_{s}(0)(X)=X\right)$. This finishes the proof.

Problem 1. Is it possible to give a similar description (cf. Theorem 6) when $\left\{l t_{0}: 0 \leq k \leq m-1\right\}$ is not a subgroup of $(G,+)$ ?

\section{Embedding a power series into a one-parameter group}

We discuss now the problem of embedding a given formal power series into a one-parameter group of formal power series. For formal power series of one variable this was considered, among others, in $[11,13]$. We give here a simple solution using the results obtained in previous sections.

Definition 1. Let $s$ be a positive integer or $s=\infty$ and let $(G,+)$ be a commutative group. A formal power series $\Phi(X) \in \Gamma^{s}$ is said to be embeddable into a one-parameter group of FPS provided there exists a one-parameter group $(F(t, X))_{t \in G}$ of FPS with $F\left(t_{0}, X\right)=\Phi(X)$ for some $t_{0} \in G$. If $G=\mathbb{K}$ then $t_{0}=1$ is assumed.

First we consider the case, when $\Phi(X) \in \Gamma_{1}^{s}$, that is $\Phi(X)=X+$ $\sum_{k=2}^{s} b_{k} X^{k}$. We will need

Lemma 9. Let $(G,+)$ be a commutative group. If the space $\mathcal{A}(G, \mathbb{K})$ of all additive functions $a: G \rightarrow \mathbb{K}$ is nontrivial, then for every $t_{0} \in G$, for which there exists $A \in \mathcal{A}(G, \mathbb{K})$ with $A\left(t_{0}\right) \neq 0$ and for every $b \in \mathbb{K}$ there exists $a \in \mathcal{A}(G, \mathbb{K})$ with $a\left(t_{0}\right)=b$.

Proof. If $b=0$, then we take $a=0$, otherwise $a=\frac{b}{A\left(t_{0}\right)} A$.

Now we are in a position to prove

Theorem 7. If the space $\mathcal{A}(G, \mathbb{K})$ is nontrivial, then every $\Phi(X)=X+$ $\sum_{k=2}^{s} b_{k} X^{k}$ can be embedded into a one-parameter group of FPS with (or without) any regularity condition. 
Proof. Fix $\Phi(X)=X+\sum_{k=2}^{s} b_{k} X^{k}$. If $\Phi(X)=X$, then we put $F(t, X)=X$ for every $t \in G$. Otherwise, let $k_{0} \in|2, s|$ be the first positive integer for which $b_{k_{0}} \neq 0$, that is $\Phi(X)=X+\sum_{k=k_{0}}^{s} b_{k} X^{k}$. By Lemma 9 we find $t_{0} \in G$ and $a \in \mathcal{A}(G, \mathbb{K})$ such that $a\left(t_{0}\right)=b_{k_{0}}$. Put $c_{k_{0}}(t)=a(t)$ (cf. (32) with $\left.p+2=k_{0}\right)$. We find a one-parameter group of FPS $F(t, X)=X+\sum_{k=k_{0}}^{s} c_{k}(t) X^{k}$ such that $F\left(t_{0}, X\right)=\Phi(X)$. In order to finish the proof it is enough to find constants $h_{j}$ for $j \in\left|k_{0}+1, s\right|$ such that (cf. (32))

$b_{n}=c_{n}\left(t_{0}\right)=h_{n} a\left(t_{0}\right)+\bar{L}_{n}^{k_{0}-2}\left(a\left(t_{0}\right) ;\left(h_{j}\right)_{j \in\left|k_{0}+1, n+1-k_{0}\right|}\right)$ for $n \in\left|k_{0}+1, s\right|$,

(we take $a_{n}=h_{n} a$ for $n \in\left|s+2-k_{0}, s\right|$ ). Note that $a\left(t_{0}\right)=b_{k_{0}}$. It is easy to see that we obtain via recurrence

$$
h_{n}=b_{k_{0}}^{-1}\left(b_{n}-\bar{L}_{n}^{k_{0}-2}\left(b_{k_{0}} ;\left(h_{j}\right)_{j \in\left|k_{0}+1, n+1-k_{0}\right|}\right)\right) \quad \text { for } n \in\left|k_{0}+1, s\right| .
$$

In the case when we are interested in some kind of regularity of the oneparameter group $(F(t, X))_{t \in \mathbb{K}}$ of FPS (in the case $G=\mathbb{K}$ ) it is enough to take $a(t)=\frac{b_{k_{0}}}{t_{0}} t$ for $t \in \mathbb{K}$.

Now, we consider the case $\Phi(X)=\sum_{k=1}^{s} b_{k} X^{k}$ with $b_{1} \notin E$. Let $\mathcal{E}(G, \mathbb{K})$ denote the set of all nonzero exponential functions $c: G \rightarrow \mathbb{K}$ and let $\mathcal{E}(G)=$ $\bigcup_{c \in \mathcal{E}(G, \mathbb{K})} c(G)$. We prove

Theorem 8. Every $\Phi(X)=\sum_{k=1}^{s} b_{k} X^{k} \in \Gamma^{s}$ with $b_{1} \in \mathcal{E}(G) \backslash E$ can be embedded into a one-parameter group of FPS with (or without) any regularity condition.

Proof. Let $c_{1} \in \mathcal{E}(G, \mathbb{K})$ be such that $c_{1}\left(t_{0}\right)=b_{1}$. We find a one-parameter group of FPS $F(t, X)=\sum_{k=1}^{s} c_{k}(t) X^{k}$ such that $F\left(t_{0}, X\right)=\Phi(X)$. This can be done by finding constants $\lambda_{n}$ for $n \in|2, s|$ such that (cf. (37))

$$
\begin{aligned}
b_{n}=c_{n}\left(t_{0}\right)= & \lambda_{n}\left(c_{1}\left(t_{0}\right)^{n}-c_{1}\left(t_{0}\right)\right)+c_{1}\left(t_{0}\right) P_{n}\left(c_{1}\left(t_{0}\right) ;\left(\lambda_{l}\right)_{l \in|2, n-1|}\right) \\
& \text { for } n \in|2, s| .
\end{aligned}
$$

Similarly as before using recurrence we get that

$$
\lambda_{n}=\left(b_{1}^{n}-b_{1}\right)^{-1}\left(b_{n}-b_{1} P_{n}\left(b_{1} ;\left(\lambda_{l}\right)_{l \in|2, n-1|}\right)\right) \quad \text { for } n \in|2, s| .
$$

Open Access. This article is distributed under the terms of the Creative Commons Attribution License which permits any use, distribution, and reproduction in any medium, provided the original author(s) and the source are credited. 


\section{References}

[1] Fejdasz, B., Wilczyński, Z.: On some $s$-1-parameter subsemigroups of the group $L_{s}^{1}$. Zeszyty Naukowe WSP W Rzeszowie 1(2), 45-50 (1990)

[2] Henrici, P.: Applied and computational complex analysis, vol. I. Power seriesintegration - conformal mapping - location of zeros. Wiley, New York (1974)

[3] Hille, E.: Ordinary differential equations in the complex domain. In: Pure and Aapplied Mathematics. Wiley, New York (1976)

[4] Jabłoński, W.: On some subsemigroups of the group $L_{s}^{1}$. Rocznik Nauk.-Dydak. WSP W Krakowie 14(189), 101-119 (1997)

[5] Jabłoński, W.: On extensibility of some homomorphisms. Rocznik Nauk.-Dydak. WSP W Krakowie 16(207), 35-43 (1999)

[6] Jabłoński, W., Reich, L.: On the solutions of the translation equation in rings of formal power series. Abh. Math. Sem. Univ. Hamburg 75, 179-201 (2005)

[7] Jabłoński, W., Reich, L.: On the form of homomorphisms into the differential group $L_{s}^{1}$ and their extensibility. Result. Math. 47, 61-68 (2005)

[8] Jabłoński, W., Reich, L.: On the standard form of the solution of the translation equation in rings of formal power series. Math. Panon. 18(2), 169-187 (2007)

[9] Jabłoński, W., Reich, L.: On homomorphisms of an abelian group into the group of invertible formal power series. Publ. Math. Debrecen 73(1-2), 25-47 (2008)

[10] Lang, S.: Algebra. Addison-Wesley, Reading (1965)

[11] Reich, L.: On the local distribution of iterable power series transformations in one indeterminate. In: Functional analysis, III (Dubrovnik, 1989), pp. 307-323. Various Publ. Ser. (Aarhus), vol. 40. Aarhus Univ., Aarhus (1992)

[12] Reich, L., Schwaiger, J.: Über analytische Iterierbatkeit formaler Potenzreihenvektoren. Österreich. Akad. Wiss. Math.-Naturwiss. Kl. S.-B. II 184(8-10), 599-617 (1975)

[13] Reich, L., Schwaiger, J.: Über einen Satz von Shl. Sternberg in der Theorie der analytischen Iterationen. uMonatshefte fr̈ Mathematik 83, 207-221 (1977)

[14] Scheinberg, S.: Power series in one variable. J. Math. Anal. Appl. 31, 321-333 (1970)

Wojciech Jabłoński

Department of Mathematics

University of Rzeszow

Rejtana $16 \mathrm{~A}$

35-310 Rzeszów

Poland

e-mail: wojciech@univ.rzeszow.pl

Ludwig Reich

Institute of Mathematics

Karl-Franzens-University Graz

Heinrichstrasse 36

8010 Graz

Austria

e-mail: ludwig.reich@kfunigraz.ac.at

Received: October 9, 2012

Revised: September 25, 2013 